\title{
Distribution and status of Paraguayan Xenarthra: towards a better understanding
}

\author{
Paul Smith $^{\mathrm{A}, \mathrm{B}, 1}$ \& Sergio D. Ríos ${ }^{\mathrm{C}, \mathrm{D}}$ \\ A FAUNA Paraguay, Encarnación, Itapúa, Paraguay. http://www.faunaparaguay.com \\ B Para La Tierra, Centro IDEAL, Mariscal Estigarribia 321 c/ Tte. Capurro, Pilar, Dpto. Ñeembucú, Paraguay. \\ http://www.paralatierra.org. E-mail: faunaparaguay@gmail.com

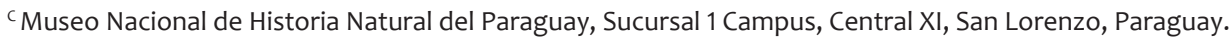 \\ E-mail: sergiord40@gmail.com \\ D Departamento de Arqueología y Paleontología, Secretaría Nacional de Cultura, Asunción, Paraguay. \\ ${ }^{1}$ Corresponding author
}

\begin{abstract}
Despite great xenarthran biodiversity, the Paraguayan armadillos and anteaters have received little attention from biologists and few data have been published about the distribution and status of the species. Furthermore, errors and contradictions persist in the literature. This paper collates specimen and literature data about this group to act as a basis for the stimulation of future research. Xenarthran specimens in all the major Paraguayan collections and all significant specimens in international collections were examined. Specimen data were collated and supplemented with literature and photographic data. Distribution data are provided according to a hierarchy of record reliability including examined specimens, non-examined specimens, literature records, photographic records, and significant observations. Thirteen species (11 armadillos and two anteaters) are confirmed to be present in Paraguay. Relevant discussion and distribution maps are provided for each species. Specimens of both Dasypus septemcinctus septemcinctus and D.s.hybridus were examined and their distribution is clarified. There is no evidence that Bradypus variegatus occurs in Paraguay. Most xenarthran specimens were collected at least 35 years ago, and the specimen record may no longer represent current distribution given the rapidly changing landscape in the country. It is concluded that available data on Paraguayan Xenarthra shows a heavy bias taxonomically towards a few species (Dasypus novemcinctus, Tolypeutes matacus, and Euphractus sexcinctus) and geographically towards the Chaco region. Distribution in the Oriental region is poorly understood, and species that are confined to that region are known from few specimens. The southern portion of the Oriental region has been virtually unsampled.
\end{abstract}

Keywords: anteaters, armadillos, Chlamyphoridae, Dasypodidae, Myrmecophagidae

Distribución y estado de los Xenarthra de Paraguay: hacia una comprensión más completa

Resumen A pesar de su riqueza en cuanto a número de especies, los armadillos y osos hormigueros del Paraguay han recibido poca atención de biólogos o naturalistas y pocos datos fueron publicados sobre la distribución y estatus de las especies. Más aún, errores y contradicciones persisten en la literatura. Este trabajo pretende reunir información de especímenes y literatura que pueda servir de base para la estimulación de futuras investigaciones sobre las especies del país. Fueron examinados especímenes de xenartros de todas las principales colecciones paraguayas y especímenes depositados en colecciones internacionales. Los datos de los ejemplares fueron cotejados y suplementados con literatura y registros fotográficos. Los datos de distribución se proveen de acuerdo a una jerarquía de confianza, incluyendo ejemplares examinados, ejemplares no examinados, registros de literatura, registros fotográficos y observaciones significativas. Trece especies (11 armadillos y dos osos hormigueros) se confirman como presentes en el Paraguay. Para cada especie se provee una discusión relevante y mapas de distribución. Fueron examinados especímenes de Dasypus septemcinctus septemcinctus y D. s. hybridus y su distribución esclarecida. No existe evidencia de que Bradypus variegatus ocurra en Paraguay. La mayor parte de los especímenes fueron colectados al menos 35 años atrás y su registro puede no representar la distribución actual de las especies, 
considerando el constante cambio de uso de la tierra en el país. Se concluye que los datos disponibles sobre xenartros paraguayos muestran un gran sesgo taxonómico hacia ciertas especies (Dasypus novemcinctus, Tolypeutes matacus y Euphractus sexcinctus) y geográfico hacia la región chaqueña. La distribución en la Región Oriental es pobremente comprendida y las especies presentes en esa región se conocen de muy pocos especímenes. La porción sureña de la Región Oriental prácticamente no ha sido muestreada.

Palabras clave: armadillos, Chlamyphoridae, Dasypodidae, Myrmecophagidae, osos hormigueros

\section{INTRODUCTION}

The Magnorder Xenarthra as traditionally understood consists of two orders: Pilosa, which includes the anteaters (Myrmecophagidae and $\mathrm{Cy}$ clopedidae) and the sloths (Megalonychidae and Bradypodidae); and Cingulata, which contains the armadillos (Dasypodidae and Chlamyphoridae) split into the four subfamilies Dasypodinae $(D a-$ sypus), Euphractinae (Chaetophractus, Euphractus, and Zaedyus), Chlamyphorinae (Chlamyphorus and Calyptophractus), and Tolypeutinae (Cabassous, Priodontes, and Tolypeutes) (Gardner, 2008; Gibb et al., 2016).

The xenarthran fauna of Paraguay is amongst the richest on the continent, with a proposed 14 species (three Dasypodidae, nine Chlamyphoridae, and two Myrmecophagidae) (Smith, 2012; de la Sancha et al., 2017; Saldívar et al., 2017). However, studies on the group in Paraguay have been few and far between, and even basic data on the distribution of many species is lacking, confused or inadequate (Smith et al., 2012; Abba et al., 2017). With a view towards consolidating the available data on the distribution and status of Paraguayan Xenarthra a review of the specimens, literature, and reliable field records was performed. The results are presented here.

\section{The history of Xenarthra studies in Paraguay}

The fauna of the Jesuit territory of "Paraquaria" (which encompassed a large area of northern Argentina, Paraguay, southern Brazil, and eastern Bolivia) were described by several early Jesuit missionaries, notably Dobrizhoffer (1784) who mentioned three armadillos, the Tatùpoyù (Euphractus sexcinctus), the Mulita (Dasypus sp.) and the Bolita (Tolypeutes matacus), as well as the Osso hormigero (Myrmecophaga tridactyla), without clarifying where he observed them. Writing around the same time Sánchez Labrador (1910, reproduced from a late $18^{\text {th }}$ Century manuscript) noted that armadillos of this region were "composed under four different names" (presumably referring to four species?), which he elected not to name, and the Bidioni (M. tridactyla).

The first systematic treatise dealing with the Paraguayan species was Azara $(1801,1802)$ who described five species of armadillos (Dasypus novemcinctus, D. hybridus, Priodontes maximus, E. sexcinctus, Cabassous tatouay) and both species of anteater (M. tridactyla and Tamandua tetradactyla). Azara $(1801,1802)$ gave only common names for his species, but two of these descriptions would later form the basis for scientific names still in use today (Dasypus hybridus and Ca. tatouay). Rengger (1830) cited the same species, noting however that he had never personally encountered either $D$. hybridus or P. maximus. Indeed, such was the lack of study in the country, that the base list for Paraguay was still derived entirely from Azara as late as Gray (1869) and Bertoni (1914), who cited the same species. Sanborn (1930) reported the first specimen of T. matacus for Paraguay, but this was overlooked by Bertoni (1939) who continued to treat the species as hypothetical.

Nomenclatural confusion in the genus Cabassous was reflected in Bertoni (1939) and he followed Yepes (1928) in listing the composite species $\mathrm{Ca}$. loricatus for Paraguay without sound basis. At this point just over half of the Paraguayan Cingulata fauna had been recorded in the country, but it was not until much later with the first explorations deep into the Chaco region that the true diversity of the country became apparent. Myers \& Wetzel (1979) added three armadillo species from the Dry Chaco (Chaetophractus villosus, C. vellerosus, and Calyptophractus retusus), and the diligent taxonomic work of Wetzel (1980) finally clarified species limits in the genus Cabassous with the description of a new species, Ca. chacoensis, from a Paraguayan type locality. The last species to be confirmed in Paraguay was Ca. unicinctus (Roguin, 1986).

The identity of the small Dasypus inhabiting Paraguay has long been the subject of debate, with these animals being alternatively referred to as D. septemcinctus or D. hybridus or both. Feijó et al. (2018) attempted to resolve this by declaring a neotype for $D$. hybridus, which they considered a subspecies of D. septemcinctus, and referring the only Paraguayan specimen available to them to the nominate D. s. septemcinctus. However, possibly based on the coordinates provided by Azara (1801, 1802) for D. hybridus, which theoretically includes the southern part of the Oriental region of Paraguay, they highlighted a potential zone of contact for the 
two subspecies that covers much of eastern and southern Paraguay. Unfortunately no specimens are available from this proposed contact zone in order to test hypotheses. Consequently, 13 species of Xenarthra (11 armadillos, 2 anteaters) are now considered to occur in Paraguay.

\section{MATERIALS AND METHOdS}

Specimens of xenarthrans from the major zoological collections in Paraguay were reviewed during 2011 to 2015, identifications were confirmed by inspection of the specimens, and locality data were collated from museum databases and specimen labels. Specimens at national park collections are also included, though these collections are uncatalogued, unregistered, and specimens are unlabelled. Data for these specimens was obtained where available by personal communication with the collectors and curators. The location of specimens in foreign museums was gleaned from the literature and from Vert Net (which returned 222 results for armadillos and 26 results for Myrmecophagidae). Where possible, specimens considered to represent significant geographical range extensions were reviewed with the assistance of museum curators. Those that did not present noteworthy distributions were assumed to be correct and not examined.

Collection codes for museums housing Paraguayan Xenarthran specimens are as follows:

ASNHC Angelo State University Natural History Collection, USA.

CBMI Colección Biológica Museo de Itaipú, Hernandarias, Paraguay.

CM Carnegie Museum of Natural History, Pittsburgh, USA.

CONN University of Connecticut Museum of Natural History, USA.

CZCEN Colección Zoológica de la FACEN, San Lorenzo, Paraguay.

CZPLT Colección Zoológica Para La Tierra, Pilar, Paraguay.

FLMNH Florida Museum of Natural History, Gainesville, USA.

FMNH Field Museum of Natural History, Chicago, USA.

MCNM Museo de Ciencias Naturales de Madrid, Spain.

MJUF Museo Jakob Unger, Filadelfia, Paraguay.

MACN Museo Argentino de Ciencias Naturales "Bernardino Rivadavia", Buenos Aires, Argentina.

MLP Museo de La Plata, Argentina.
MHNG Musée d'Histoire Naturelle de Genève, Switzerland.

MNC Museo Nacional de Chile, Santiago, Chile (currently the Museo Nacional de Historia Natural de Chile).

MNHNP Museo Nacional de Historia Natural del Paraguay, San Lorenzo, Paraguay.

MSB Museum of Southwestern Biology, Albuquerque, USA.

MTD Museum für Tierkunde, Dresden, Germany.

MZ Museo Zoológico Universidad Nacional de Asunción, Agronomía y Veterinaria, San Lorenzo, Paraguay (currently the Museo Schade SCH).

MZB Museu de Ciències Naturals de Barcelona, Spain.

MZS Musée Zoologique de l'Université et de la Ville, Strasbourg, France.

NHM Natural History Museum, London, United Kingdom.

NHMB Naturhistorisches Museum Basel, Switzerland.

RBINS Royal Belgian Institute of Natural Sciences, Belgium.

ROM Royal Ontario Museum, Ontario, Canada.

$\mathrm{SCH}$ Museo Schade, Facultad de Ciencias Agrarias, San Lorenzo, Paraguay.

TTU Museum of Texas Tech University, Texas, USA.

UAM University of Alaska Museum, USA.

UMZC University Museum of Zoology, Cambridge, United Kingdom.

USNM National Museum of Natural History, Smithsonian Institute, Washington, USA.

SMNH Swedish Museum of Natural History, Stockholm, Sweden.

ZFMK Zoologisches Forschungsmuseum Alexander Koenig, Bonn, Germany.

Species accounts begin with the current common name, scientific name, and author following Bertassoni (2018), McDonough \& Loughry (2018), and Superina \& Abba (2018) with one taxonomic change proposed by Feijó et al. (2018). The original described name, author, and type locality follow. There then follows a referenced list of the synonyms used in the Paraguayan literature with a (hopefully self-explanatory) single word descriptor of the subject of the publication, as follows: conservation, diet, distribution, ecology, dictionary, ethnography, genetics, guide, habitat, list, mention, parasitology, records, specimen/s, taxonomy, tracks, and use. 


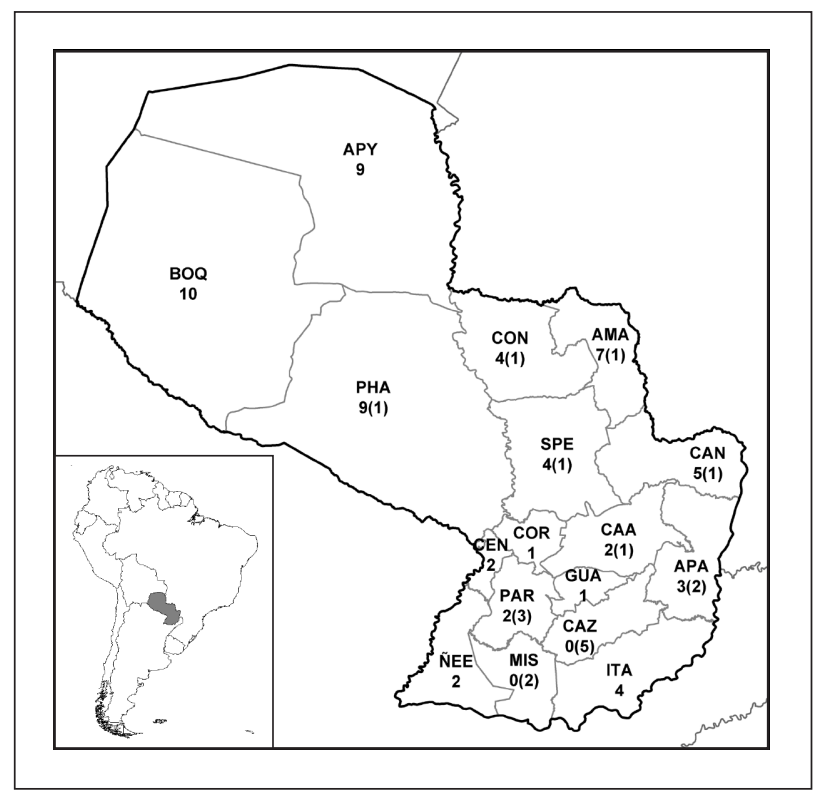

FIGURE 1. Xenarthran biodiversity by department. NumBer represents species documented with specimens, NUMBER IN PARENTHESES additional species with reliable reports documented in this paper but with no voucher specimen. AMA: Amambay; APA: Alto Paraná; APY: Alto Paraguay; BOQ: Boquerón; CAA: Caaguazú; CAN: Canindeyú; CAZ: Caazapá; CEN: Central; CON: Concecpción; COR: Cordillera; GUA: Guairá; ITA: Itapúa; MIS: Misiones; Ñ̃E: Ñeembucú; PAR: Paraguarí; PHA: Presidente Hayes; SPE: San Pedro.

The synonymy deals only with Paraguayan literature or literature citing Paraguayan specimens and is not intended to be a complete list of synonymy for the species.

Local names: Local common names published in the Paraguayan literature are provided. An attempt to reference the earliest published usage for each name is made.

Comments: Addressing noteworthy or confusing themes in the Paraguayan literature.

There then follows a "hierarchical reliability" approach to the Paraguayan distribution of each species. This approach is taken so as to not unduly bias understanding by depending solely on the limited specimen record. The hierarchies are, in order of documented reliability: 1) examined specimen, 2) specimen not examined, 3) published literature record, 4) published photographic record, 5) reliable field observation by one of the authors or knowledgeable local observer. The vast majority of the photographic records referred to are available online at http://www.faunaparaguay.com and can be found using their unique photo code preceded with FPMAMM. Records are presented with the political department in bold capitals, followed by the details of the record (in alphabetical order; FIG. 1). For specimen records this involves the specimen number followed by the locality. These records are also mapped distinguishing the hierarchical categories so that readers may interpret their reliability for themselves (FIGs. 3-15). Records corresponding to categories 4) published photographic records and 5) reliable field observation include only localities that are not covered by any one of the previous three categories. A full gazeteer of the localities mentioned in the text is provided as SUPPLEMENTARY MATERIAL on the Edentata website (http://www. xenarthrans.org/newsletter/Smith\&Rios_Supplementary_material.pdf).

The criteria for inclusion of literature was that it was published in Paraguay or specifically deals with Paraguay, or in the case of international publications, that it makes specific reference to Paraguayan specimens. Every effort was made to be thorough in this regard, though undoubtedly some obscure references will have been missed.

The conservation status for all Paraguayan species was assessed by Smith (2012) and Abba et al. (2017) and is not repeated here. Detailed compiled reviews of the ecology of all species are provided by Smith (2007a-f, 2008a-f, 2009, 2011) and these data are also not repeated here. However, the distribution information that is provided here should be understood as replacing the Paraguayan distribution data provided in those works.

A statement on the ecological affinities of each species in Paraguay is provided based on the ecoregions defined by Olson et al. (2001) (FIG. 2), and later updated by Guyra Paraguay (2005) and Mereles (2013) (no map shapes currently available). These can be broadly defined as follows: Alto Paraná Atlantic Forest (subtropical humid forests of eastern Paraguay); Cerrado (central South American savanna of northern eastern Paraguay); Dry Chaco (low, arid thorn forest and scrub of the western Occidental region); Humid Chaco (palm savanna and marshlands of the Paraguay River Basin); Pantanal (gallery forests and swamps of the north-eastern Chaco); Cerrados del Chaco (an area of Cerrado in the northern Chaco contiguous with the Chiquitania of Bolivia) and Southern Cone Mesopotamian Savanna (flooded Mesopotamian grasslands of the southern Oriental region) (FIG. 2).

\section{RESULTS}

Locality data for 13 species and 14 subspecies of Xenarthra in four families is provided (FIG. 2) and one species is rejected. Distributions for each of the confirmed species are mapped (FIGS. 3-15) and confidence levels are distinguished from each other in the map keys, allowing future researchers to make their own decisions on reliability of records consistent with the demands of their own research. 


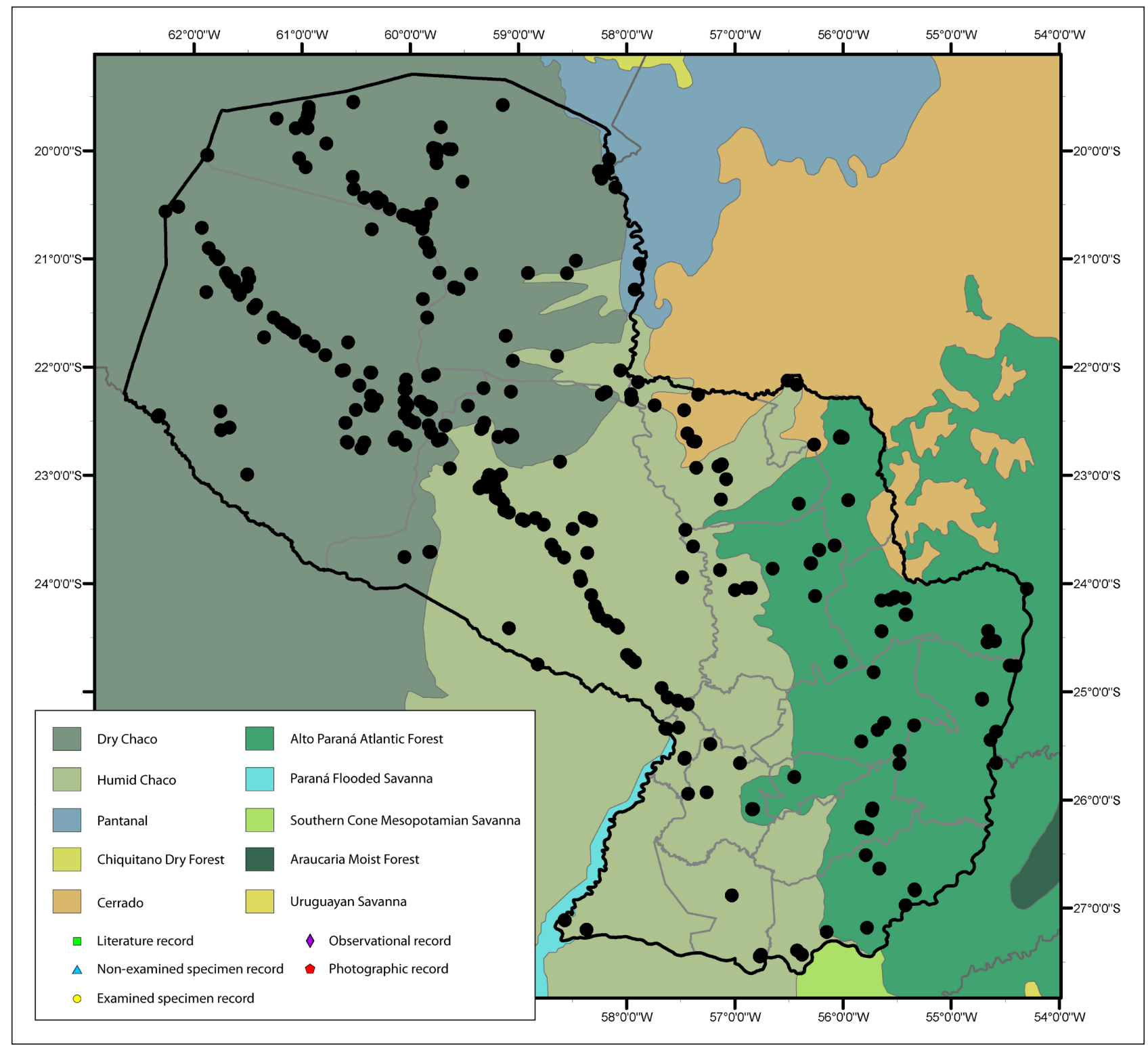

FIGURE 2. Geographical coverage of all the localities cited in this paper on a base map of the regional ecoregions, and with key to symbols used on species maps.

\section{DASYPODIDAE: Long-nosed armadillos}

\section{SEVEN-BANDEd ARMADILLO}

Dasypus septemcinctus

(Linnaeus, 1758) (FIG. 3)

[Dasypus] septemcinctus Linnaeus 1758:51. Type locality "in Indiis" corrected to "Brasilia" by Erxleben (1777) and restricted to Pernambuco, Brazil by Cabrera (1958).

lor[icatus] hybridus Desmarest 1804:28. No type locality given. Based on Azara (1801). Restricted to San Ignacio, Misiones, Paraguay by Cabrera (1958), neotype designated by Feijó et al. (2018) from Estancia Jeffries, eight miles East of Treinta y Tres, department of Treinta y Tres, Uruguay.

Dasypus hibridus Rengger (1830: ecology).
Dasypus hybridus Rengger (1830: ecology); Bertoni (1939: list); Cabrera (1958: taxonomy); Wetzel \& Mondolfi (1979: taxonomy); Gamarra de Fox \& Martin (1996: records); Neris et al. (2002: distribution); Smith (2008f: ecology); Smith (2012: conservation).

Tatusia hybrida Bertoni (1914: list).

Dasypus septemcinctus Masi Pallarés \& Benítez Usher (1982: parasitology); Gamarra de Fox \& Martin (1996: no records); Gamarra de Fox et al. (1998: no records); Yahnke et al. (1998: distribution); Villalba \& Yanosky (2000: tracks); Esquivel (2001: guide); Neris et al. (2002: distribution); Nava et al. (2007: parasitology); Abba \& Vizcaíno (2008: specimens); Smith (2008e: ecology); Smith (2012: conservation). 


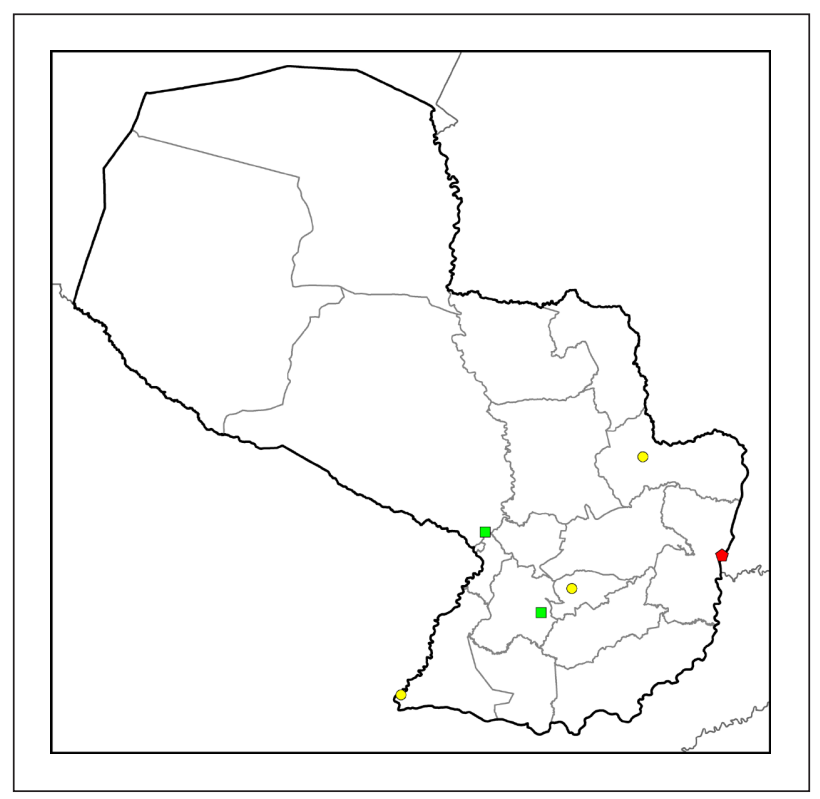

FIGURE 3. Distribution of Dasypus septemcinctus in Paraguay.

Dasypus hybridis Yahnke et al. (1998: distribution).

Dasypus sp. Fariña \& Hostettler (2003: distribution).

Tolypeutes matacus Fariña \& Hostettler (2003: distribution).

Dasypus septencinctus Masi Pallarés (2011: ecology).

Dasypus septemcinctus hybridus Feijó et al. (2018: taxonomy).

Dasypus septemcinctus septemcinctus Feijó et al. (2018: taxonomy).

Local names: Aché: Tatu kuju (Fariña \& Hostettler, 2003); Guaraní: Tatú-mburicá (Azara, 1802); Tatu-mburica (Rengger, 1830); Tatu Hu (Gamarra de Fox et al., 1998); Tatu Hu'i (Neris et al., 2002); Tatu bolita, Tatu'i (Fariña \& Hostettler, 2003); Spanish: Mulita (Azara, 1802); Mulita orejuda, Armadillo siete bandas, Mulita de las pampas (Gamarra de Fox \& Martin, 1996); Mulita común (Neris et al., 2002); Armadillo (Morales, 2007).

Comments: Following persistent confusion between D. septemcinctus and D. hybridus (e.g., Gray, 1874; Lönnberg, 1928), the first attempt to elucidate their identities was by Hamlett (1939) who restricted the range of D. septemcinctus to Brazil. He noted that it was replaced by D. hybridus in Rio Grande do Sul and southern Mato Grosso, the states that immediately border Paraguay. Despite this subsequently becoming the taxonomic orthodoxy, D. septemcinctus continued to figure frequently in Paraguayan publications dealing with armadillos since then (e.g., Yahnke et al., 1998; Neris et al., 2002; Smith, 2012), as well as in significant taxonomic works (e.g., Wilson \& Reeder, 2005; Wetzel et al., 2008) even though none specifically referenced
Paraguayan specimens. This issue was recognized by Gamarra de Fox \& Martin (1996) and Gamarra de Fox et al. (1998) who listed the species as one with "problems with records" for Paraguay.

Feijó et al. (2018) reviewed the global taxonomy of the genus Dasypus, and considered D. hybridus to be a subspecies of $D$. septemcinctus, identifiying one "small Dasypus" specimen available from Paraguay (MNHNP 3365, collected on 7 December 2006 by Ismael Mora) as D. septemcinctus, but not examining any additional specimens.

The subspecies D. s. hybridus was first associated with Paraguay on the basis of Azara $(1801,1802)$. Azara noted that the Guaraní name Tatú-mburicá means "mule armadillo", presumably in reference to the long, mule-like ears (and this may also be the source of the scientific name hybridus-Feijó et al. (2018)). Azara stated that he had "never found the species north of 26.5 degrees", leading Cabrera (1958) to fix the type locality of D. hybridus as San Ignacio, Paraguay (Misiones department) on the basis that it represented approximately the northern limits of the range mentioned by Azara (1801, 1802). However, the area covered by Azara's work was much greater than the boundaries of modern-day Paraguay and the latitude given also covers large areas of Argentina and Brazil, rendering Cabrera's restriction somewhat arbitrary. It is often overlooked that in the introduction to his armadillos Azara (1802) stated that his fourth, sixth, seventh, and eighth armadillo species (D. hybridus is the sixth) were distributed in the "southern part" of his geographic area (approximating to the River Plate basin) and "not in Paraguay", though he added that "in truth the sixth can be seen only in the most southerly part of the province" (Paraguay) (Azara, 1802:105). Given that the Paraguay of Azara's time included the now Argentine provinces of Misiones and Formosa, it is by no means clear that he was referring to an area north of the Paraná River, in modern-day Paraguay. Though the latitude he provided does include a portion of the southern part of the Oriental region of Paraguay, it also perhaps importantly includes substantial areas of Formosa and Misiones, Argentina (Abba \& Superina, 2016).

Gray (1869) (who never visited Paraguay) described D. hybridus as "very common" in Paraguay, but this was not an opinion shared by Rengger (1830) (who lived in Paraguay for many years) who said he searched for the species "in vain". Azara's statement about the great abundance of the species clearly referred to the "austral part" of the "La Plata province" rather than the northern reaches that incorporate modern Paraguay.

Feijó et al. (2018) declared a neotype (FMNH 29334) from Estancia Jeffries, eight miles East of Treinta y Tres, department of Treinta y Tres, Uruguay 


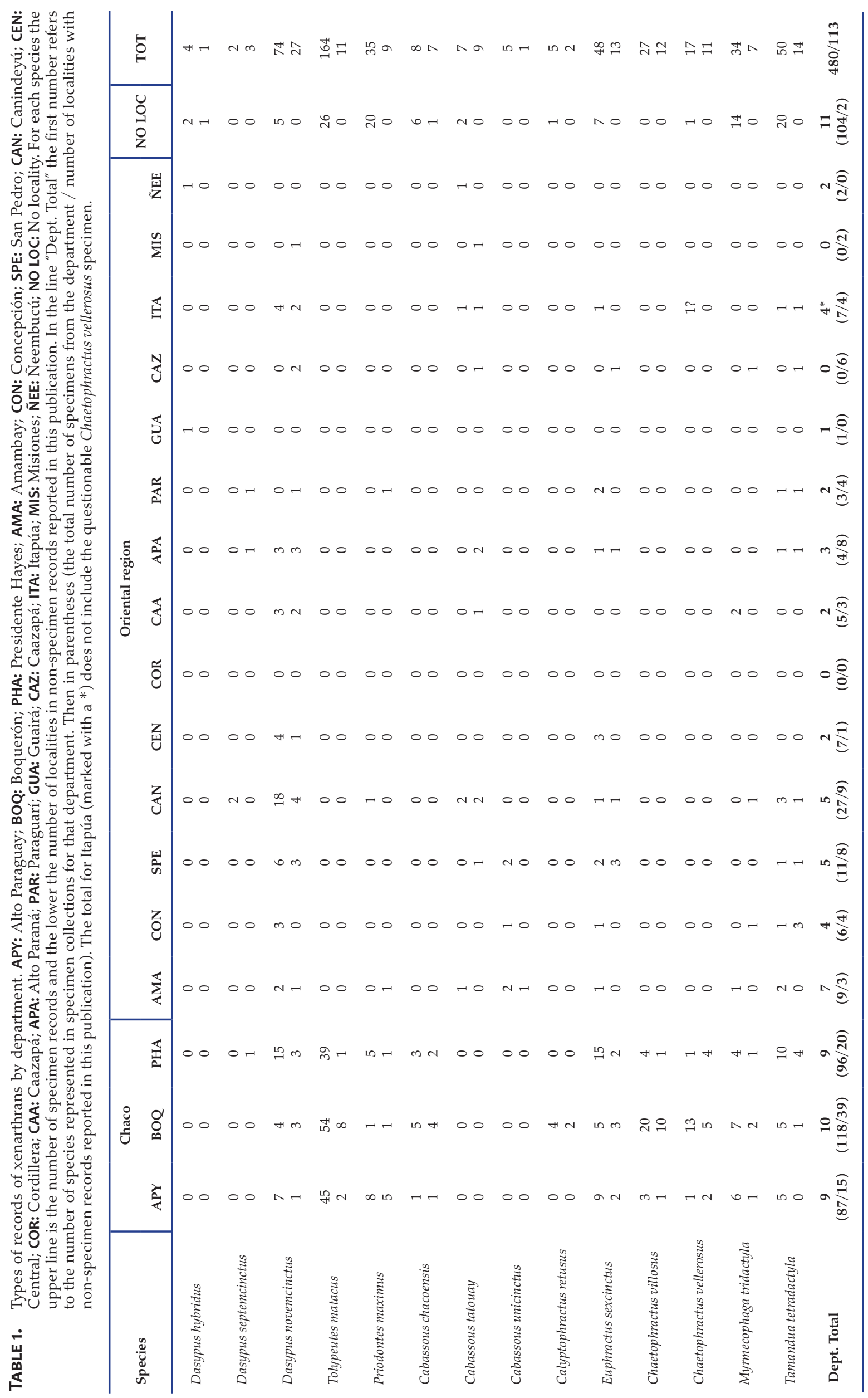


collected on December 1, 1926 by Colin Campbell Sanborn.

A mounted specimen in the Royal Ontario Museum (ROM 9508130010) was bequeathed to the museum by Toronto University, which in turn received the material from a now defunct and unidentified educational institution. The institution had purchased the specimen from "Gerrard's of London", a company well known for inexact localities. Consequently this specimen may or may not have originated in Paraguay.

An individual from the Mbaracayú Reserve, Canindeyú department was identified as D. septemcinctus "based on measurements" (Hugo del Castillo, pers. comm.). The specimen was captured between 10-14 November 2000 by Aché indigenous hunters who referred to it as "tatu bolita", a common name usually associated with the Chaco species T. matacus (and possibly contributing to baseless reports of that species in eastern Paraguay; e.g., Fariña \& Hostettler, 2003). Although the specimen was apparently collected, its current whereabouts are unknown.

Specimen MACN 28.226 identified in the museum catalogue as D. septemcinctus is a badly stuffed juvenile specimen with skull included from "Puerto Guarany" collected by Juan B. Daguerre (Abba \& Vizcaíno, 2008). The specimen lacks a tail, appears to lack at least one movable band and has been poorly repaired. We suspect it is likely a juvenile $D$. novemcinctus, but consider it unidentifiable. Daguerre is known to have collected in this area during at least August and September 1928, but two specimens of D. novemcinctus in the MACN $(28.224,28.225)$ also bear the exact same collection data. Consequently, we consider the identification and data accompanying this specimen to be suspect.

Skull specimen MVZ 4824, which appears in the museum catalogue as D. septemcinctus and on the specimen label as Dasypus sexcinctus, is actually referable to $D$. novemcinctus on the basis of size and the presence of eight teeth in each tooth row.

We attribute the specimen from Canindeyú (MNHNP 3365) and the photographs from Alto Paraná (FPMAMM 1174-1175) to D. s. septemcinctus, and the specimens from Guairá (ZMB 40472, bought by the museum in December 1927 from J. Flemming and illustrated at FPMAMM 1179) and Ñeembucú (NHMB 1450, collected on 27 June 1904 by Carl Ternetz and illustrated at FPMAMM 1176-1178) to D. s. hybridus based on morphometrics (notably ratio of head length to ear length). The limited information on Paraguayan specimens suggests that $D$. s. septemcinctus occurs at very low density in a restricted area of the eastern Oriental region of Paraguay at the interface of the Cerrado and Atlantic Forest ecoregions, and it is replaced in the grasslands and Humid Chaco of the southwestern Oriental region by D. s. hybridus. However, the extent to which these populations contact in central Paraguay is unknown. There is no physical evidence to support the literature citations for the presence of "D. hybridus" in Paraguarí or Presidente Hayes departments, and these may represent range extrapolations or identification errors (Masi Pallarés \& Benítez Usher (1982), for example, contains several serious nomenclatural errors). We recommend that they be treated with caution pending further data.

Specimens examined: "Paraguay" MZS 01598 (Wetzel \& Mondolfi, 1979), ROM 9508130010; Canindeyú: "Estancia La Rama III, 2409.426'S, $55^{\circ} 34.299^{\prime} W^{\prime \prime}$ MNHNP 3365; Guairá: Villarrica ZMB 40472 (Wetzel \& Mondolfi, 1979); Ñeembucú: Curupayty NHMB 1450 (Wetzel \& Mondolfi, 1979).

Specimens not examined: None.

Literature citations: "South of $26.5^{\circ} \mathrm{S}$ (Azara 1801, 1802); Paraguarí: "Parque Nacional Ybycuí" (Yahnke et al., 1998); Presidente Hayes: "Villa Hayes" (Masi Pallarés \& Benítez Usher, 1982).

Photographic documentation: Alto Paraná: Refugio Biológico Tati Yupí FPMAM1174-1175 (Nelson Pérez in FAUNA Paraguay, 2018).

\section{NiNE-BANDED ARMADILLO \\ Dasypus novemcinctus \\ Linnaeus, 1758 (FIG. 4)}

[Dasypus] novemcinctus Linnaeus 1758:51. Type locality "in America Meridionali" restricted to Pernambuco, Brazil by Cabrera (1958).

Dasypus novemcinctus Rengger (1830: ecology); Bertoni (1939: list); Wetzel \& Mondolfi (1979: taxonomy); Masi Pallarés \& Benítez Usher (1982: parasitology); Servicio Forestal Nacional (1982: guide); Roguin (1986: distribution); Fujita et al. (1994: parasitology); Fujita et al. (1995: parasitology); Gamarra de Fox \& Martin (1996: records); Lowen et al. (1996: distribution); Gamarra de Fox et al. (1998: conservation); Yahnke et al. (1998: distribution); Villalba \& Yanosky (2000: tracks); Areskoug (2001: habitat); Esquivel (2001: guide); Neris \& Colman (2001: guide); Frutos \& Van den Bussche (2002: genetics); Neris et al. (2002: distribution); Fariña \& Hostettler (2003: distribution); Hill et al. (2003: use); Neris \& Franco Rivarola (2005: guide); Yeo et al. (2005: parasitology); Cartes (2007: use); Nava et al. (2007: parasitology); Smith (2007c: ecology); Meritt (2008: ecology); Ramírez Pinto \& Velázquez (2010, distribution); Masi Pallarés (2011: ecology); Smith (2012: conservation); Acosta \& López (2013: parasitology); Centrón et al. (2013: use); 


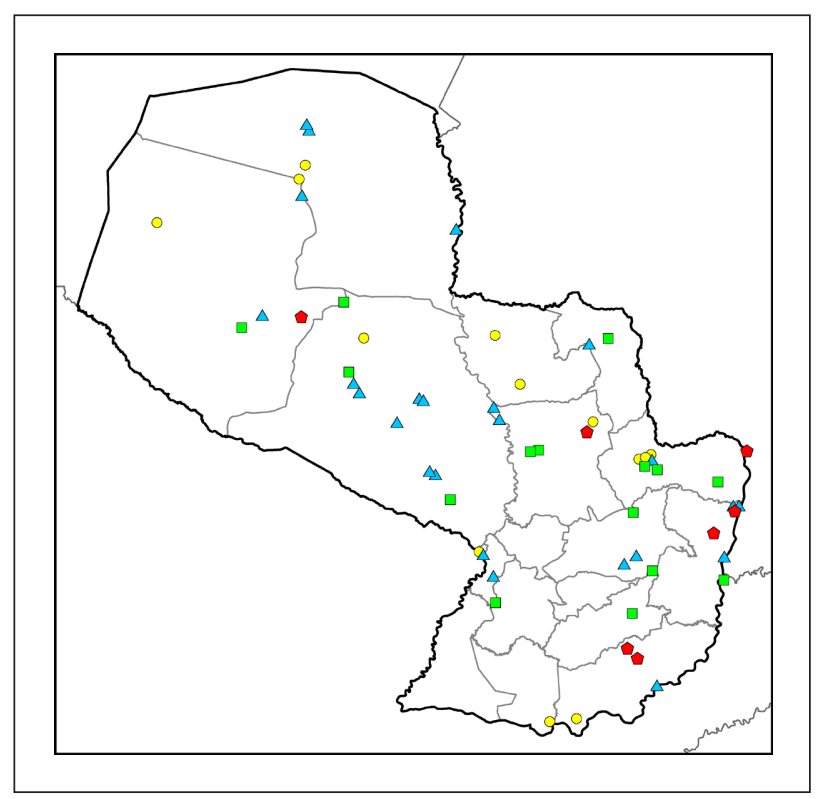

FIGURE 4. Distribution of Dasypus novemcinctus in Paraguay.

Velázquez \& Ramírez Pinto (2014: guide); Epp (2018: guide).

Tatusia novemcincta Bertoni (1914; list).

Dasypus novemcinctus novemcinctus Cabrera (1958: taxonomy).

Dasypus novecinctus Gatti (1985: dictionary).

Local names: Aché: Tatu kuju (Esquivel, 2001); Tatu (Fariña \& Hostettler, 2003); Ayoreo: Ajamei (Neris \& Colman, 2001); Guaraní: Tatuhú (Azara, 1802); Tatu-hu (Rengger, 1830); Tatú-hû (Bertoni, 1914); Tatu hu (Servicio Forestal Nacional, 1982); Tatú-eté, Tatujú (Gatti, 1985); Tatu hû (Velázquez \& Ramírez Pinto, 2014); Spanish: Quirquincho negro (Azara, 1802); Armadillo negro (Servicio Forestal Nacional, 1982); Mulita grande (Gamarra de Fox \& Martin, 1996); Armadillo (Esquivel, 2001); Mulita de nueve bandas (Velázquez \& Ramírez Pinto, 2014).

Comments: The species was first cited for Paraguay by Azara (1801) citing the common name Tatuhú, meaning "black armadillo". The species occurs throughout the country in all the ecoregions.

Two stuffed specimens on display at the Museo de la Entidad Binacional Yacyretá, Ayolas, Misiones department lack collection data but may have been collected locally as the species is known to occur in this area.

A female specimen MNHNP 543 (Ruta Transchaco $0.5 \mathrm{~km}$ S Fortín Teniente Enciso) is notably smaller and with lower scute counts for the cephalic shield and fourth movable band than other adult Paraguayan specimens in the MNHNP. The measurements and counts are consistent with the description of Dasypus mazzai Yepes, 1933 and geographically the specimen comes from an area where D. mazzai might perhaps be most likely to occur should it be present in Paraguay. However, the specimen was catalogued as a juvenile (perhaps on the basis of small size?) and no skeletal material is available to confirm or dismiss that assertion, except for a mandible. Given that the morphological characteristics that define $D$. mazzai are unclear (they overlap almost entirely with $D$. novemcinctus) and that no genetic material is available for this specimen, for the time being we maintain the identification as $D$. novemcinctus pending additional material, but alert future researchers to the possibility of D. mazzai occurring in this area of Paraguay.

Specimens examined: Mounted specimen lacking data MJUF; "Paraguay" MVZ 4824; Alto Paraguay: "58 km SO de Agua Dulce" MNHNP 542; Boquerón: "4 km south Madrejón, admin. Parque Nacional Defensores del Chaco" MNHNP 541; "Ruta Transchaco 0.5 km S Fortín Teniente Enciso" MNHNP 543; Canindeyú: no specific locality MNHNP 2038; "Jejui” SCH 810; “Mbaracayú Wildlife Reserve 2 km NE central camp $24^{\circ} 07^{\prime} \mathrm{S}, 57^{\circ} 55^{\prime} \mathrm{W}^{\prime \prime} \mathrm{MNHNP} 917$; "Reserva Mbaracayú" MNHNP 2032, 2033, 2040, 2042, 2043, 2045; Central: "Cerro Lambaré, 25²0.517'S, 57³8.404'W" MNHNP 3366; Concepción: "Estancia Cerrito" MNHNP 2507; "Parque Nacional Serranía San Luís, $22^{\circ} 37.43^{\prime}$ S 57 $21.28^{\prime} W^{\prime \prime}$ MNHNP 2506; "Rio Apa" SCH 52; Itapúa: skull lacking data at Pro Cosara museum; MACN 25173 "Isla Yacyretá, Barranquita"; "Reserva Natural Yacyreta" skeleton lacking data on display at park headquarters. Presidente Hayes: "Reserva Laguna Porá" MNHNP 1188; San Pedro: "Estancia Las Mañanitas" CZPLT 220, 221, 224, 225.

Specimens not examined: "Paraguay" MACN 9.2, 29.209; 43.064 (Abba \& Vizcaíno, 2008); Alto Paraná: "Refugio Fauna" CBMI 155; "Puerto Sauce" CBMI 0005; "Refugio Biológico Tati Yupi, Hernandarias" CBMI 0088; Alto Paraguay: no specific locality FLMNH 20653, 20654; "67 km by road N Fortín Madrejón" UMMZ 126293; "120 km E of Mayor Pablo Lagarenza, W of Aguadulce" CONN 19910; "Puerto Guarany" MACN 28.224, 28.225 (Abba \& Vizcaíno, 2008); Amambay: "28 km SW Pedro Juan Caballero" UMMZ 125246, 125247; Boquerón: "Fortín Toledo" MTD 24981 (Ziegler et al., 2002); "Teniente Martínez, 175 km N Filadelfia" MSB 54421; Caaguazú: "3 km N of Campo 9" MSB 135356; "Estancia Primera, Guayraungua River" (= Río Guyrauguá) MCZ 30943, 30944; Canindeyú: no specific locality UAM 46587, 46588, 46590, 46591, 46604, 46605; "Reserva Natural del Bosque Mbaracayú" TTU 80202, 80206, UAM 46589; Central: "Colonia Nueva Italia" FMNH 54354, 54355; Lambaré ZMB 85906; Itapúa: “1 km N of Rio Paraná, E of Rio Pirapó" CONN 16894; Presidente Hayes: "85 km E Loma Plata, Laguna Pora" CONN 19959; 
"211 km NW (by road) Villa Hayes" MVZ 145362; "Chaco Experimental Station $295 \mathrm{~km}$ NW (by road) Villa Hayes" CONN 15760, 15761, MVZ 145363, 145364; "km 96 Transchaco" CONN 17517; "km 150 rte Transchaco" MNHG 1690.090; "km 159 rte Transchaco" MNHG 1689.090 (Roguin, 1986); "km 305 Transchaco" CONN 17333; "Estancia Deolinda" SMNH 600182; "Waikthlatingmayalwa" UMZC E954, E955 E956; San Pedro: "11 km S (by air) of Concepción, E bank of Rio Paraguay" $23^{\circ} 30^{\prime} S$, 57²9'W" UMMZ 166715; "Puerto Ybapobo" FMNH 26649 .

Literature citations: Alto Paraguay: "Casanillo" (Frutos \& Van den Bussche, 2002); Alto Paraná: "Puerto Bertoni" (Bertoni, 1914; 1939); Amambay: "Parque Nacional Cerro Corá" (Lowen et al., 1996); Boquerón: "Gran Siete" (Areskoug, 2001); Caaguazú: no specific locality (Masi Pallarés \& Benítez Usher, 1982); "Estancia Kaa'gua Rory" (Lowen et al., 1996); Caazapá: "Estancia Golondrina-Caazapa" (Frutos \& Van den Bussche, 2002); "Parque Nacional Caaguazú" (Lowen et al., 1996); Canindeyú: "Mbaracayú Reserve" (Hill et al., 2003); "Reserva Natural Privada Itabó" (Lowen et al., 1996); "Reserva de Patrimonio Aché de Kuetuvy" (Centrón et al., 2013); Central: "Asunción" (Bertoni, 1939); Presidente Hayes: "Estancia Juan de Salazar" (Frutos \& Van den Bussche, 2002); "Ruta Transchaco km 106" (Frutos \& Van den Bussche, 2002); Paraguarí: "Lago Ypoa" (Frutos \& Van den Bussche, 2002); San Pedro: "Chamorro Cué outskirts of San Pedro de Ycuamandyyú" (Fujita et al., 1995); "Patiño outskirts of San Pedro de Ycuamandyyú" (Fujita et al., 1995).

Photographic documentation: Alto Paraná: "Reserva Itabó Itaipú" FPMAM69 (Paul Smith in FAUNA Paraguay, 2018); "Reserva Limoy Itaipú"

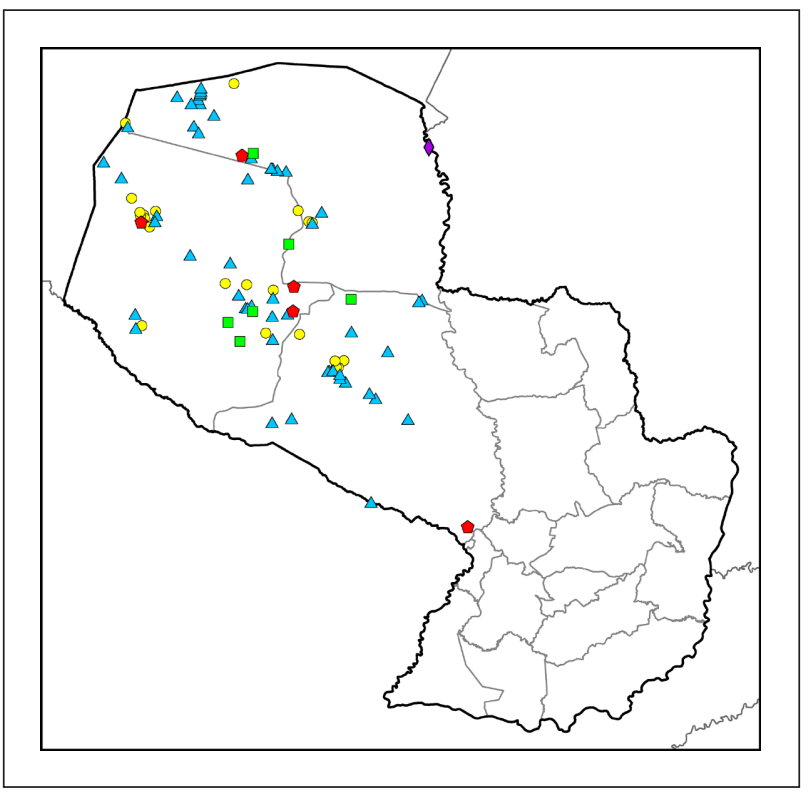

FIGURE 5. Distribution of Tolypeutes matacus in Paraguay.
FPMAM1165 (Paul Smith in FAUNA Paraguay, 2018); Boquerón: "Loma Plata" FPMAM71 (Paul Smith in FAUNA Paraguay, 2018); Canindeyú: "Reserva Mbaracayú Itaipú" FPMAM887 (Paul Smith in FAUNA Paraguay, 2018); Itapúa: "Estancia Nueva Gambach" FPMAM68, 70, 72-76 (Pro Cosara in FAUNA Paraguay, 2018); "Reserva Kanguery" FPMAM895-896 (Paul Smith in FAUNA Paraguay, 2018); San Pedro: "Reserva Natural Laguna Blanca" (PLT camera trap photo 2 December 2011).

Reliable observations: Boquerón: "Tunacojai Indigenous Reserve" (Paul Smith); Misiones: "Ñu Guazu" (fide Arne Lesterhuis); Presidente Hayes: "Laguna Capitán" (Paul Smith).

\section{CHLAMYPHORIDAE: TOLYPEUTINAE: Tolypeutine armadillos \\ SOUthern THREe-BANDEd ARMAdillo Tolypeutes matacus \\ (Desmarest, 1804) (FIG. 5)}

Lor[icatus]. matacus Desmarest 1804:28. No locality mentioned. Based on Azara (1801). Sanborn (1930) restricted the type locality to Tucumán, Argentina.

Tolypeutes matacus Sanborn (1930: distribution, taxonomy); Cabrera (1958: taxonomy); Wetzel \& Lovett (1974: specimens); Wetzel (1985b: distribution); Roguin (1986: distribution); Brooks (1995: distribution); Fujita et al. (1995: parasitology); Gamarra de Fox \& Martin (1996: records); Gamarra de Fox et al. (1998: conservation); Yahnke et al. (1998: distribution); Villalba \& Yanosky (2000: tracks); Areskoug (2001: habitat); Neris \& Colman (2001: guide); Neris et al. (2002: distribution); Fast Schartner (2004: guide); Neris \& Franco Rivarola (2005: guide); Yeo et al. (2005: parasitology); Cartes (2007: use); Smales (2007: parasitology); Smith (2007f: ecology); Abba \& Vizcaíno (2008: specimens); Meritt (2008: ecology); Vinke \& Vinke (2008: popular); Ramírez Pinto \& Velázquez (2010: distribution); Smith (2012: conservation); Acosta \& López (2013: parasitology).

Tolypeustes matacos Bertoni (1939: list); Gatti (1985: dictionary).

Tolypeustes Seelwische (1980: ethnography).

Tolypeutes mataco Masi Pallarés \& Benítez Usher (1982: parasitology); Chase Sardi (1983: ethnography); Nava et al. (2007: parasitology).

Tolypeutes macatus Smales (2007: parasitology).

Local names: Ayoreo: Auco (Neris \& Colman, 2001); Enhlet: Yalva (Fast Schartner, 2004); Guaraní: Tatu bolita (Azara, 1802); Tatú-bolito, Tatú-apará (Wetzel \& Lovett, 1974); Tatú bolita (Gamarra de Fox \& Martin, 1996); Tutú bolita (Morales, 2007); Mennonite dialect: Bauljirtillschwien 
(Fast Schartner, 2004); Nivaclé: C'acjo (Seelwische, 1980); Spanish: Bolita (Dobrizhoffer, 1784); Matáco (Azara, 1802); Tatú naranja (Chase Sardi, 1983); Armadillo (Gamarra de Fox et al., 1998); Mataco (Gamarra de Fox \& Martin, 1996); Quirquincho bola (Neris \& Colman, 2001).

Comments: Dobrizhoffer (1784) described the "Bolita" from the Paraquaria region, noting that it was capable of rolling itself into a ball, but Azara (1802) specifically stated that the species did not occur in Paraguay. The earliest citation we can find affirming the species presence in Paraguay is Sanborn (1930) who mentions a specimen collected by Alexander Wetmore " $110 \mathrm{~km}$ west of Puerto Pinasco" (USNM 236356). According to his own notes, Wetmore collected at this locality on 23 September 1920 (Wetmore, 1926). However, there are earlier specimens: UMZC E1184, a foetus with placenta collected during 1907 by W.E. Agar in an unspecificed locality in the Paraguayan Chaco, and three undated partial specimens (UMZC E1181, E1182, E1183) collected by Kerr at Waikthlatingmayalwa, Presidente Hayes department. According to his writings Kerr was at this locality from late October 1896 until mid-February 1897 and again in May 1897 (Kerr, 1901). Bertoni (1939), apparently unaware of these reports, listed the species as hypothetical in the Paraguayan Chaco.

A carapace of T. matacus in the Pro Cosara museum at Estancia Nueva Gambach, Itapúa department was not collected locally and originated from an unspecified locality in the Dry Chaco of Boquerón department.

The specimens in the MNHNG cited by Smales (2007) with inventory numbers only (39690, 39691, 39692) are extremely geographically confused. The locality "Estancia La Conquista close to Fortín Pratt Gill" is not "in Central Province" as stated but in Boquerón department. These records refer to the three specimens (MNHG 1689,061; 1689,062; 1689,063) published in Roguin (1986), two of which were collected at Estancia La Conquista on 27 July 1985 and the other on 26 July 1985 "15 km west of Pratts Gill" according to the museum catalogue. Roguin's (1986) text description of this locality as "15 km Fortín Boquerón direction de Pratts Gill" creates confusion with Fortín Boquerón on the border of Boquerón and Presidente Hayes departments.

The Tolypeutes sp. listed by Smales (2007) for "Caazepa [sic] Province" refers to Ca. unicinctus MNHG 1875,003 (see species description below) and was actually collected in Concepción department. There is no evidence that T. matacus occurs east of the Río Paraguay in Paraguay. Tolypeutes matacus is confined to the Occidental region of Paraguay, occuring in the Dry Chaco, Humid Chaco, and Cerrados del Chaco ecoregions, and marginally in the Pantanal.
Specimens examined: Alcohol specimens lacking data in MNHNP with field numbers Z1, Z3; "Chaco" SCH 359, 699, 1818; Carapace lacking data MJUF; Alto Paraguay: "Destacamento Militar Gabino Mendoza" uncatalogued specimens in MNHNP with field numbers TK 65701, TK 65745; "Estancia Tres Marias 21⒖81.5'S, 59³5.59' 'W" MNHNP 2505; "Estancia Tres Marias $21^{\circ} 16.82^{\prime} \mathrm{S}, 59^{\circ} 32.89^{\prime} \mathrm{W}^{\prime \prime}$ MNHNP 2504; "Palmar de las Islas $19^{\circ} 39.11^{\prime} \mathrm{S}, 60^{\circ} 35.88^{\prime} \mathrm{W}^{\prime}$ MNHNP 3367; Boquerón: "Approx. $2 \mathrm{~km}$ norte de la casa de admin, PN Tte Enciso" MNHNP 511; “5 km N Cruce de los Pioneros" uncatalogued alcohol specimen in MNHNP with field number SF02; "15 km al sur de la casa de la admin por la picada nueva de la Empresa de Madelon, PN Tte Enciso" MNHNP 512; "24.4 km E Mariscal Estigarribia, $22^{\circ} 02.85 S 60^{\circ} 21.93 W^{\prime \prime}$ uncatalogued specimen in MNHNP with field number TK 60234; "25 km N of Filadelfia" MNHNP 2894; "Approx. $30 \mathrm{~km} \mathrm{NO}$ casa de control, PN Tte Enciso sobre la Transchaco" MNHNP 514; "Approx 150 km North of Filadelfia sobre la ruta" MNHNP 510; "Base Aeronautica Pratts Gill" MNHNP 1654; "Mariscal Estigarribia" MNHNP 1655; "Newland" (= Neuland) MNHNP 1895, 1896; "PN Tte Enciso" MNHNP 834; "PN Tte Enciso $21^{\circ} 08.20^{\prime} \mathrm{S}, 61^{\circ} 30.17^{\prime} \mathrm{W}^{\prime}$ MNHNP 3369; "PN Tte Enciso $21^{\circ} 11.12^{\prime} \mathrm{S}, 61^{\circ} 41.27^{\prime} \mathrm{W}^{\prime}$ MNHNP 3368; "PN Tte Enciso" MSB 54420 two carapaces lacking data at park headquarters; "Ruta Transchaco $8 \mathrm{~km}$ north, casa de admin., PN Tte Enciso" MNHNP 513; Presidente Hayes: "Estancia Salazar, Buey" uncatalogued alcohol specimen in MNHNP with field number SFUH23; "Estancia Salazar, Galpón" uncatalogued alcohol specimens in MNHNP with field number SFUH28, SFUH29; "Estancia Salazar, Represa Valente (?)" uncatalogued alcohol specimen in MNHNP with field number SFUH24; "Estancia Salazar, Retiro $3^{\circ}$ tramo" uncatalogued alcohol specimen in MNHNP with field number SFUH4, SFUH17, SFUH18; "Estancia Salazar, Romualdo Cue" uncatalogued alcohol specimens in MNHNP with field numbers SFUH8, SFUH9, SFUH27; "Estancia Salazar, Tajamar Tereré" uncatalogued specimen in MNHNP with field number SFUH3; "Estancia J. Salazar, 5 km N Embarcadero" uncatalogued specimen in MNHNP with field number SFUH1.

Specimens not examined: "Paraguay" ASNHC 12916, CONN 16652, MACN 13.782, 27.147 (Abba \& Vizcaíno, 2008), RBINS 386137, 386138, 391699, 391700, 502640, 502641, 502642, 502643, 569741, 569742, 688821, 688822, 699124, 699125, 699126, 699127; "Paraguayan Chaco" UMZC E1184; Alto Paraguay: " $8 \mathrm{~km} \mathrm{~N}$ and $37 \mathrm{~km} \mathrm{~W}$ of Mayor Pablo Lagerenza" CONN 19890; "18 km by rd W Fortín Madrejón" UMMZ 124718; "28 km N and 52 km W of Mayor Pablo Lagerenza" CONN 19884, 19885, 20484; "30 km W of Puerto Casado along railroad" FMNH 54353; “39 km WNW Mayor Pablo Lagarenza” CONN 19868, 19869; “41 km WNW Mayor Pablo 
Lagarenza” CONN 19871; "42 km WNW Mayor Pablo Lagarenza and 6 km N” CONN 19873; “42 km WNW Mayor Pablo Lagarenza and 8 km N" CONN 19874; “42 km WNW Mayor Pablo Lagarenza and 11 km N" CONN 19883; “42 km WNW Mayor Pablo Lagarenza and 16 km N" CONN 19942, 19943; "45 km WNW Mayor Pablo Lagarenza" CONN 19872; "50 km WNW Madrejón, Misión Nuevo Tribu" UMMZ 124719, 125621, 126292; “Capitán Pablo Lagerenza" TTU 79709; "Estancia Tres Marías" TTU 79817, 79819, 79820; “Gabino Mendoza" TTU 79721; “Laguna Placenta” TTU 79876, 79878, 79879; "Madrejón" CONN 19892; "Road to Fortín Lagarenza-i" TTU 79911, 79912; "Road to Puerto Casado" TTU 79913; "Palmar de las Islas" TTU 79881, 79883, 79884, 79885, 79886, 79887, 79907, 79908, 79909, 79910; Boquerón: "10 km south of Filadelfia" TTU 79951; “11.8 km by rd W Fortín Madrejón” UMMZ 124721; "15 km Fortín Boquerón direction de Pratts Gill” MNHG 1689.061 (Roguin, 1986); “20.1 km by rd W Fortín Madrejón" UMMZ 124720; “30km N Mariscal Estigarribia" TTU 79953; "40 km NW of Pratt-Gill A.F. Base" CONN 19893; "50 km WSW of Fortín Madrejón" AMNH-M-248394; “474km (by road) NW Villa Hayes" MVZ 145365; "km 483 Transchaco" CONN 18313; "km 590 Transchaco" CONN 18311; “km 764 Transchaco” CONN 17608; "Estancia Iparoma, 19 km N Filadelfia" CONN 19817, 19818, 19897, 19904, 19905, 19906, 19907, 19909, 19913, 19914, 19915, 19916, 19917, 19918; "Estancia La Conquista, Pratts Gill" MNHG 1689.062, 1689.063 (Roguin, 1986); "Estancia Madelon, 15 km S Tte Enciso park HQ" FLMNH 20652; "Filadelfia, $37 \mathrm{~km} \mathrm{~W}$; Estancia Toledo-Molineros" FMNH 164098; "Fortín Toledo" MTD 24890 (Ziegler et al., 2002); "Guachalla, Rio Pilcomayo" FMNH 54405; "Loma Plata Mennonite Colony" KU 92664; "Nueva Asunción" FLMNH 20652; "Teniente Enciso" CONN 17513, 17520, 18142; Presidente Hayes: "1.5 km W of line camp, Juan de Zalazar" CONN 16637; “4 km W of line camp, Juan de Zalazar" CONN 16649; " $4 \mathrm{~km}$ S of Rio Verde, $5 \mathrm{~km}$ E of Trans-Chaco" CONN 16650; " $5 \mathrm{~km}$ NE of line Camp, on Rio Verde; Juan de Zalazar" CONN 16743; "5 km NE Pozo Colorado" CONN 17518; "10 km E of Nuevo Retiro Aloncito" MSB 57376, 57399; "km 305 Transchaco, Retiro" CONN 19797; "Laguna Pora, 85 km E Loma Plata" CONN 19919, 19920, 19820, 20485, 20486; "Chaco Experimental Station 295 km NW (by road) Villa Hayes" MVZ 145366, 145367; "Estancia Elsita" SMNH 603568; "Estancia Hermosa, Rio Siete Puntas, 100 km W Concepción" SMNH 603503, 603509, 603526, 603528; "General Bruguez" CBMI 0245; "Puerto Pinasco, 110km W" USNM 236356; "Transchaco -23.351, -58.737" MNHG 1701.100; "Vicinity of km 300; Juan de Zalazar" CONN 16882; "Waikthlatingmayalwa" UMZC E1181, E1182, E1183.
Literature citations: Alto Paraguay: "Cerro León"

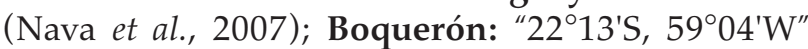
(Ziegler et al., 2002); "22 ${ }^{\circ} 45^{\prime} \mathrm{S}, 6^{\circ} 27^{\prime} \mathrm{W}^{\prime}$ (Ziegler et al., 2002); "Gran Siete" (Areskoug, 2001); "surroundings of $21^{\circ} 21-39^{\prime} \mathrm{S}, 5^{\circ} 51-54$ 'W (Ziegler et al., 2002); "Toledo" (Nava et al., 2007); Presidente Hayes: "Juan de Salazar" (Wetzel, 1985b).

Photographic documentation: Alto Paraguay: "PN Defensores del Chaco" FPMAM94 (Sjeff Ollers in FAUNA Paraguay, 2018); Boquerón: "Loma Plata" FPMAM1105 (Paul Smith in FAUNA Paraguay, 2018); "Parque Nacional Teniente Enciso" FPMAM95 (Marcelo Bombaci in FAUNA Paraguay, 2018); FPMAM96-100 (Paul Smith in FAUNA Paraguay, 2018); "Tunakojai Indigenous Reserve" FPMAM916-917 (Paul Smith in FAUNA Paraguay, 2018); Presidente Hayes: "Estancia Golondrina" FPMAM1187PH (Sergio D. Ríos in FAUNA Paraguay, 2018).

Reliable observations: Alto Paraguay: "Puerto 14 de Mayo-Karcha Bahlut" (Sergio D. Ríos July 2015).

\section{GIANT ARMADILLO}

Priodontes maximus

(Kerr, 1792) (FIG. 6)

Dasypus maximus Kerr 1792:112. Type locality "Cayenne", French Guiana.

Dasypus giganteus Rengger (1830: ecology).

Priodontes giganteus Cabrera (1912: specimen); Cabrera (1958: taxonomy); (Masi Pallarés 2011: ecology).

Prionodontes gigas Bertoni (1914: list).

Prionodontes giganteus Bertoni (1939: list); Meritt (1973: records); Gatti (1985: dictionary).

Priodontes maximus Wetzel \& Lovett (1974: specimens); Gamarra de Fox \& Martin (1996: records); Gamarra de Fox et al. (1998: conservation); Yahnke et al. (1998: distribution); Villalba \& Yanosky (2000: tracks); Esquivel (2001: guide); Neris et al. (2002: distribution); Fariña \& Hostettler (2003: distribution); Neris \& Franco Rivarola (2005: guide); Cartes (2007: use); Smith (2007b: ecology); Abba \& Vizcaíno (2008: specimens); Meritt (2008: ecology); Smith (2012: conservation); Weiler \& Nuñez (2012: distribution, conservation); GAT \& OPIT (2014: ethnography).

Local names: Aché: Kry'y pura vachu (Esquivel, 2001); Kry'y pura (Fariña \& Hostettler, 2003); Ayoreo: Jochacai (GAT \& OPIT, 2014); Guaraní: Tatú-wasú (Bertoni, 1914); Tatú-asú, Tatú-guasú (Gatti, 1985); Tatú guazú (Gamarra de Fox \& Martin, 1996); Tatu guasu (Gamarra de Fox et al., 1998); Nivaclé: Pôlhenjataj (Seelwische, 1980); Spanish: 


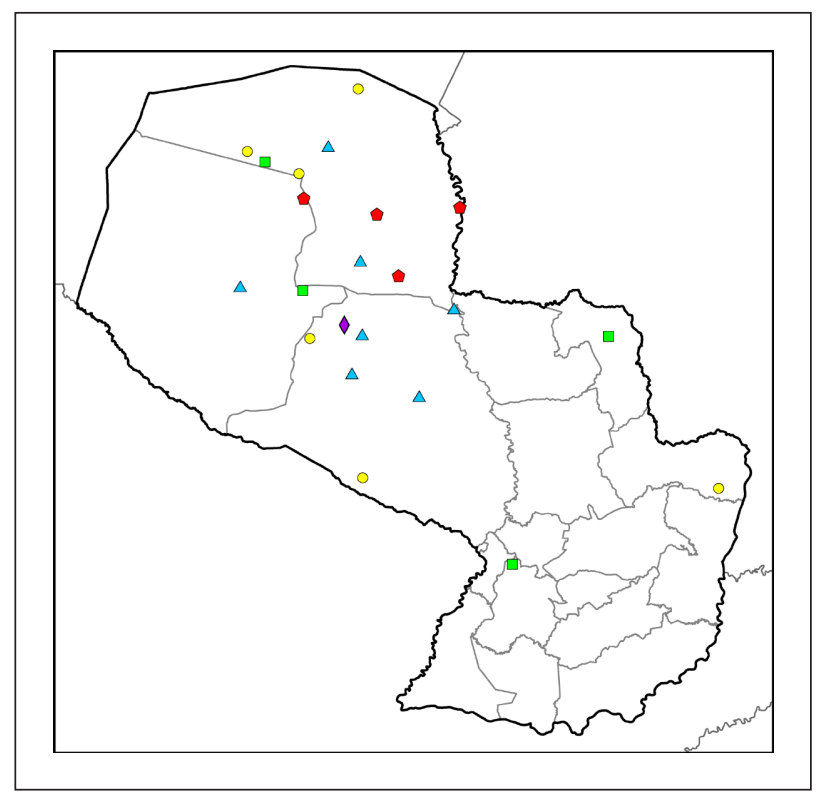

FIGURE 6. Distribution of Priodontes maximus in Paraguay.

Tatú negro grande de bosque (Azara, 1802); Tatu carreta (Bertoni, 1914); Tatú-carreta (Gatti, 1985); Tatú carreta (Gamarra de Fox \& Martin, 1996); Armadillo gigante (Gamarra de Fox et al., 1998).

Comments: First cited for Paraguay by Azara $(1801,1802)$ who called it "Máximo" noting that the species did not have a common name and referring to it as "very scarce". Azara (1802) stated that the species was found only in "the great forests of the north of Paraguay" and this was echoed by Rengger (1830) who stated that the species could be found only in northeastern Paraguay in "lands inhabited only by a few Guaraní". These references were presumably the basis of Bertoni's $(1914,1939)$ statement that the species "still exists in the north of Paraguay". Sánchez-Labrador (1910) makes a possibly veiled $18^{\text {th }}$ Century reference to this species when he alludes to a species of armadillo "the size of a medium-sized dog" that was hunted and roasted by the indigenous "Mbayá" and which "feeds many". Mbayá was the pejorative name given by the indigenous Guaraní to the indigenous Guaycurú (today known as the Caduveo) of the northern part of the Oriental region of Paraguay and adjacent Mato Grosso do Sul.

An illustration in Cabrera (1912) purports to show an Azara specimen of this species from "Paraguay" (MCNM 504). Cabrera (1958) restricted the type locality of the name Priodontes giganteus to Pirayú, Paraguay on the basis of Azara (1801, 1802).

Bertoni stated that his friend "Prof. Dr. Anisits had a live specimen". Dr. Anisits was a well-known Hungarian professor who resided in Asunción (Benítez, 1986), so this statement unfortunately sheds little light on the origin of the specimen.
The only specimen we could trace from the Oriental region of Paraguay is CBMI 0037 from Pozuelo, Canindeyú department supposedly collected on 19 July 1979. The species is frequently listed for the Reserva Natural Bosque Mbaracayú in Canindeyú department (Esquivel, 2001; Fariña \& Hostettler, 2003) but there is no documentation of its occurrence in the reserve and no solid reports of its existence there could be traced. A carapace on display at the Museo de la Entidad Binacional Yacyretá, Ayolas, Misiones department lacks collection data and probably did not originate locally.

Weiler \& Nuñez (2012) list additional localities for the species in Boquerón and Alto Paraguay departments based on records of burrows, but these are omitted here as secondary evidence of occurrence. The core of the Paraguayan range is now the Dry Chaco and Cerrados del Chaco ecoregions west of the Paraguay River, the species being close to extinction in the Cerrado ecoregion of eastern Paraguay (Smith, 2012).

Specimens examined: Mounted specimen and carapace lacking data MJUF; "Chaco" SCH 2398; "Paraguay" MACN 9.1, 10.21, 11.41, 13.23, MCNM 504 (Cabrera, 1912); Alto Paraguay: "Chovoreka" MNHNP 2011; "Fortín Madrejón" MNHNP 1170; "PN Defensores del Chaco" MNHNP 820; Canindeyú: "Pozuelo" CBMI 0037; Presidente Hayes: "Estancia Sofía" MNHNP 1126; "Ruta Trans-Chaco km 379" MNHNP 1075.

Specimens not examined: "Paraguay" AMNH-M42697, NHM 1978.3255, FLMNH 72913, MACN 13.80 (Abba \& Vizcaíno, 2008); "Paraguay, Zoo Buenos Aires" MACN 11.14, 13.68 (Abba \& Vizcaíno, 2008); Alto Paraguay: "144 km NE Filadelfia" CONN 19507, 19926; "Colonia San Miguel Arcángel, Distrito de Fuerte Olimpo" CZCEN 059, 060; "Puerto Casado" MACN 45.029 (Abba \& Vizcaíno, 2008); Boquerón: "Mariscal Estigarribia" CONN 18310; Presidente Hayes: "85 km E Loma Plata, Laguna Pora" CONN 19957; "Estancia Deolinda" SMNH 622705; "Rio Verde, Estancia Kent" CONN 15978 (Wetzel \& Lovett, 1974).

Literature citations: Alto Paraguay: "surroundings of $20^{\circ} 29^{\prime} \mathrm{S}, 6^{\circ} 18^{\prime} \mathrm{W}^{\prime \prime}$ (Ziegler et al., 2002); Amambay: "Parque Nacional Cerro Corá" (Yahnke et al., 1998); Boquerón: "Area of Mariscal Estigarribia" (Meritt, 1973); "Colonia Ayoreo Campo Loro" (Weiler \& Nuñez, 2012); Paraguarí: "Pirayúu" (Azara, 1801, 1802).

Photographic documentation: Alto Paraguay: "approximately $35 \mathrm{~km} \mathrm{~S}$ of Madrejón by road" roadkill 13 October 2017 FPMAM1180-1185 (Paul Smith in FAUNA Paraguay, 2018); "near Fuerte Olimpo" FPMAM92 (Arne Lesterhuis in FAUNA Paraguay, 2018); "Estancia Beatriz close to Cruce Paragro" 


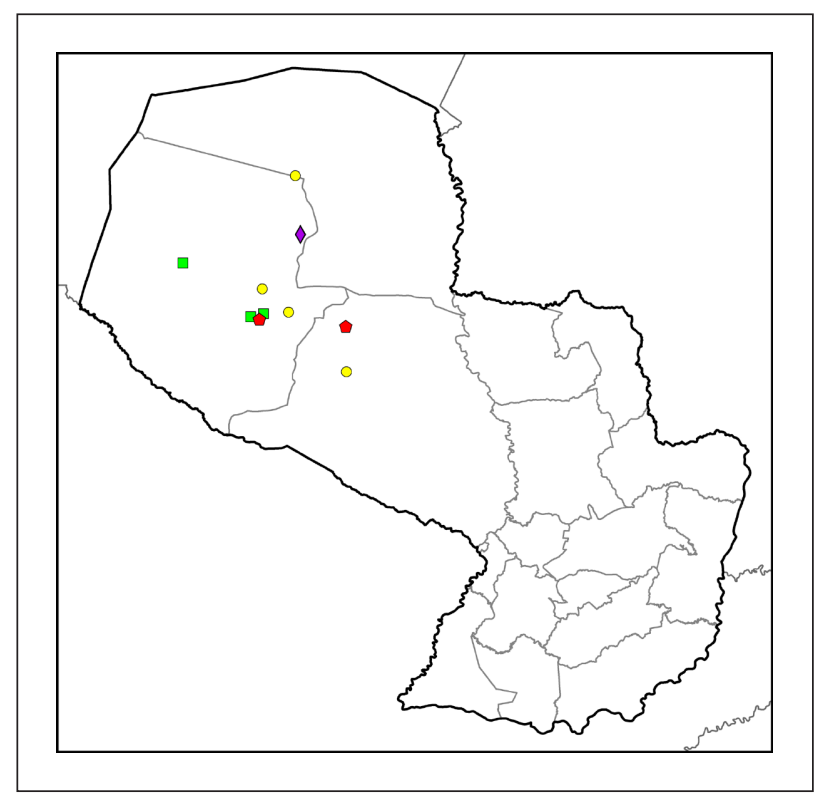

FIGURE 7. Distribution of Cabassous chacoensis in Paraguay.

roadkill 13 July 2012 FPMAM1158 (Hugo del Castillo in FAUNA Paraguay, 2018); "Estancia Morocha" roadkill 19 July 2002 FPMAM93 (Hugo del Castillo in FAUNA Paraguay, 2018).

Reliable observations: Presidente Hayes: "Chaco Lodge" (fide Norbert Epp).

\section{Chaco Naked-tailed Armadillo \\ Cabassous chacoensis \\ Wetzel, 1980 (FIG. 7)}

Cabassous chacoensis Wetzel 1980:335 Type locality "5-7 km W of Est. Juan de Zalazar, Presidente Hayes department, Paraguay".

Cabassous chacoensis Wetzel (1980: taxonomy); Wetzel (1985b: distribution); Gamarra de Fox \& Martin (1996: records); Gamarra de Fox et al. (1998: conservation); Neris et al. (2002: distribution); Neris \& Franco Rivarola (2005: guide); Abba \& Vizcaíno (2008: specimens); Meritt (2008: ecology); Vinke \& Vinke (2008: ecology); Smith (2009: ecology); Hutterer \& Peters (2010: specimens); Smith et al. (2011: mention); Smith (2012: conservation); GAT \& OPIT (2014: ethnography).

Cabossus chacoensis Yahnke et al. (1998: distribution).

Local names: Ayoreo: Ogode (GAT \& OPIT, 2014); Guaraní: Tatú'ai menoré (Gamarra de Fox \& Martin, 1996); Tatu'ai menore (Gamarra de Fox et al., 1998); Tatu A'i (Neris et al., 2002); Spanish: Tatú de rabo molle (Gamarra de Fox \& Martin, 1996); Armadillo (Gamarra de Fox et al., 1998); Armadillo chaqueño de cola desnuda (Morales, 2007); Cabasú chico (Vinke \& Vinke, 2008).
Comments: Described on the basis of Paraguayan specimens (Wetzel, 1980). Contra Roguin (1986), Ca. loricatus of Bertoni (1939) does not refer to this species. Bertoni (1939), who was unfamiliar with the Chaco mastofauna, cites loricatus for Paraguay fide Yepes. However, the only pre-1939 reference to loricatus in Paraguay by that author is Yepes (1928) in an unannotated checklist employing numerous synonyms as valid species. His distribution of "Guyanas, Paraguay, Brasil" demonstrates that the name is not applicable to chacoensis and, as he cites no specimens, it is clearly a composite of different species. Cabassous loricatus sensu Yepes (1935) is a synonym of $C a$. chacoensis, but no reference is made to the species in Paraguay in that work and its contents are clearly different to those of Yepes (1928). The Ca. loricatus of Cabrera (1958) that mentions the Paraguayan Chaco in the distribution is again a composite of at least two species according to the distribution provided. The first reference to this species in Paraguay is thus its description by Wetzel (1980).

The known Paraguayan range is restricted to the central Chaco, an area of transition between Dry and Humid Chaco.

Specimens examined: Mounted specimen lacking data MJUF (same as FPMAM915); alcohol specimen lacking data in MNHNP; Boquerón: "Philadelfia" (= Filadelfia) uncatalogued specimen in MNHNP with field number SFZ01.

Specimens not examined: "Paraguay" MLP843, MLP16.IX.35.86, MLP16.IX.35.85 (Abba \& Vizcaíno, 2008); "Chaco" MZ 1600 (Wetzel, 1980); Alto

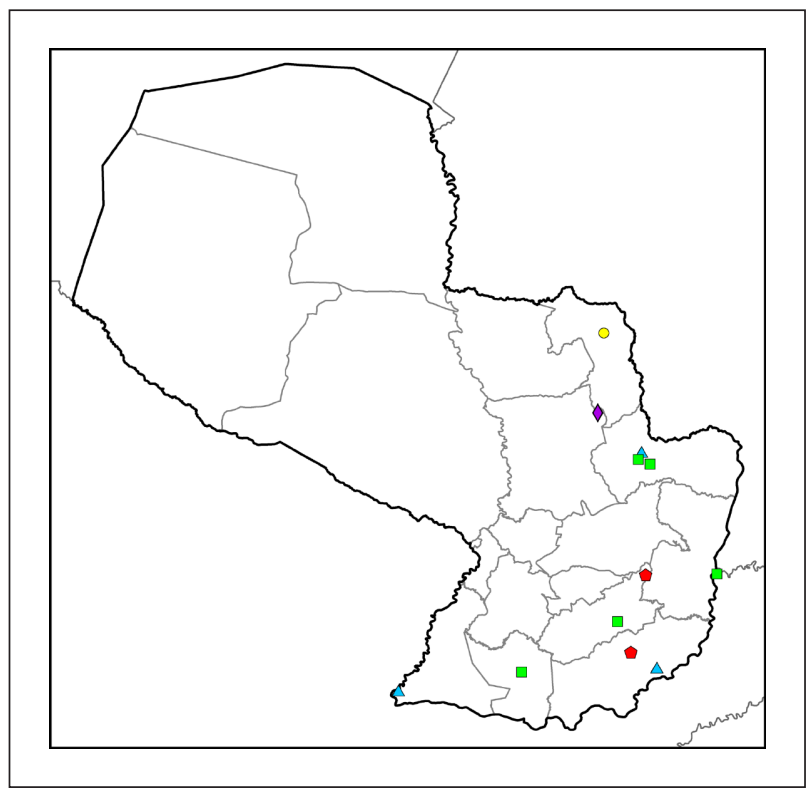

FIGURE 8. Distribution of Cabassous tatouay in Paraguay. 
Paraguay: "Estancia Toro 1-Madrejón" TTU 79816; Boquerón: “27 km S Madrejón” FLMNH 20650; “Filadelfia” USNM 531004, ZFMK 60.317 (Wetzel, 1980; Hutterer \& Peters, 2010); “26.5 km east of Mariscal Estigarribia" TTU 79952; Presidente Hayes: "5-7 km W Juan de Zalazar" CM 67067 (holotype), CONN 16891, 16892 (Wetzel, 1980; Hutterer \& Peters, 2010).

Literature citations: Alto Paraguay: "Campo Loa area in front of Estancia Amistad" December 2010 (Sabine \& Thomas Vinke in DVD Paraguay Salvaje 1, Filadelfia, Paraguay); Boquerón: "area around Toledo" (= Fortín Toledo) (Meritt, 2008); "Estancia Mariposa" (Vinke \& Vinke, 2008) though locality not specified in the text; Presidente Hayes: "Juan de Zalazar" (Wetzel, 1985b).

Photographic documentation: Jakob Unger Museum, Filadelfia FPMAM915 (Paul Smith in FAUNA Paraguay, 2018); Boquerón: "Fortín Toledo" FPMAM81-82 newborns 30 June 2009 (Hugo del Castillo in FAUNA Paraguay, 2018); Presidente Hayes: "Chaco Lodge" (fide Joe Sarvary/Para La Tierra).

Reliable observations: Boquerón: "Estancia 120 $\mathrm{km}$ north of Filadelfia near Picada Ancha $22^{\circ} 27^{\prime} \mathrm{S}$, 6046'W" 25 February 2012 (fide Sabine \& Thomas Vinke).

\section{GREATER NAKED-TAILEd ARMADILLO Cabassous tatouay \\ (Desmarest, 1804) (FIG. 8)}

Loricatus tatouay Desmarest 1804:28. No type locality given, but restricted to Paraguay $27^{\circ}$ latitude south by Cabrera (1958) who considered the description to be based on Azara (1801).

Dasypus gymnurus Rengger (1830: ecology).

Lysiurus unicinctus Bertoni (1914: list).

Cabassous unicinctus Bertoni (1939: list).

Cabassous tatouay Cabrera (1958: taxonomy); Wetzel (1980: taxonomy); Gamarra de Fox \& Martin (1996: records); Esquivel (2001: guide); Neris et al. (2002: distribution); Fariña \& Hostettler (2003: distribution); Hill et al. (2003: use); Neris \& Franco Rivarola (2005: guide); Abba \& Vizcaíno (2008: specimens); Smith (2008c: ecology); Smith et al. (2011: mention); Smith (2012: conservation); Velázquez \& Ramírez Pinto (2014: guide).

Cabassous tatouai Gamarra de Fox et al. (1998: conservation).

Cabossus tatouay Yahnke et al. (1998: distribution).

Cabassus tatouay Centrón et al. (2013: use).

Local names: Aché: Kru'y (Esquivel, 2001); Tatú vai (Centrón et al., 2013); Guaraní: Tatuaí (Azara, 1802); Tatu-ay (Rengger, 1830); Tatú' ai (Gamarra de Fox \& Martin, 1996); Tatu ai (Esquivel, 2001); Tatu A'i (Neris et al., 2002); Tatu-ai (Velázquez \& Ramírez Pinto, 2014); Spanish: Armadillo grande (Esquivel, 2001); Armadillo (Neris et al., 2002); Armadillo cola desnuda (Morales, 2007); Cabasú grande, Tatú de rabo molle (Velázquez \& Ramírez Pinto, 2014).

Comments: The species was first cited for Paraguay by Azara (1801) who was unsure as to the origin of the common name, speculating that Tatuaí meaning "warty armadillo" was unfitting of the animal and that it may have been a shortening of Taturaí or "naked armadillo" in reference to the tail. Azara's text was based on a description provided to him by his friend the Jesuit priest Pedro Blás Noséda who "received an individual close to his town", modern day San Ignacio, Misiones department.

This species occurs at low density in Cerrado, Atlantic Forest and Mesopotamian Grasslands in eastern Paraguay.

Specimens examined: Amambay: "near Cerro Corá" MNHNP uncatalogued, field number TK61367.

Specimens not examined: "Paraguay" MLP16. IX.35.84; "Paraguay" MACN 9.4. (Yepes, 1935); Canindeyú: "Reserva Mbaracayúu" (= Reserva Bosque Mbaracayú) MNHNP 2049 lost?; no specific locality UAM 46602; Itapúa: "Capitán Meza" MACN 47.377 (Wetzel, 1980; Abba \& Vizcaíno, 2008); Neembucú: "Curupaity" (= Curupayty) NHMB (Wetzel, 1980).

Literature citations: Alto Paraná: "Puerto Bertoni" (Bertoni, 1914; 1939); Caazapá: "Reserva Natural Tapytá" (Velázquez \& Ramírez Pinto, 2014); Canindeyú: "Reserva Bosque Mbaracayúu" (Hill et al., 2003; Cartes, 2007); "Reserva de Patrimonio Aché de Kuetuvy" (Centrón et al., 2013); Misiones: "town of my friend Noséda around 270" (= San Ignacio) (Azara, 1801, 1802).

Photographic documentation: Caaguazú: "Reserva Natural Privada Ypeti" FPMAM90-91 between 17 and 20 June 1997 (José Luís Cartes in FAUNA Paraguay, 2018); Itapúa: "Estancia Nueva Gambach" approximately 2003 FPMAM1159 (Hans Hostettler in FAUNA Paraguay, 2018).

Reliable observations: San Pedro: "Yaguarete Forest" roadkill between 5 and 14 August 2000 (fide Hugo del Castillo).

\section{SOUTHERN NAKED-TAILED ARMADILLO Cabassous unicinctus (Linnaeus, 1758) (FIG. 9)}

Dasypus unicinctus Linnaeus 1758:50. Type locality "Africa", but restricted to Surinam by O. Thomas (1911).

Cabassous unicinctus squamicaudis Roguin (1986: distribution); Smith et al. (2011: distribution). 


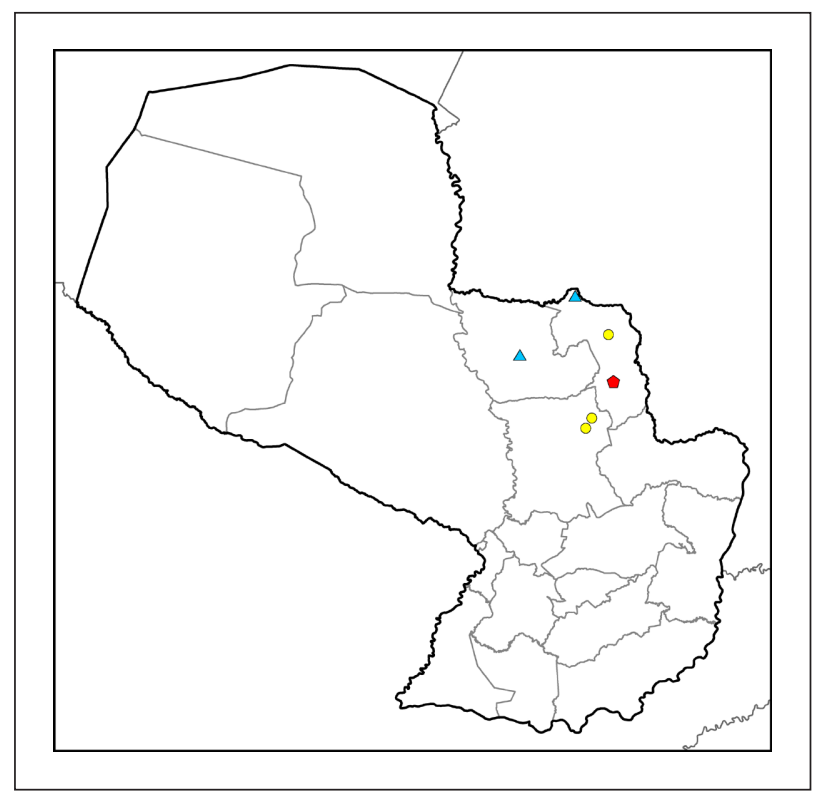

FIGURE 9. Distribution of Cabassous unicinctus in Paraguay.

Tolypeutes sp. Smales (2007: parasitology).

Cabassous unicinctus (Masi Pallarés 2011: ecology); Smith (2011: ecology); Smith (2012: conservation).

Local names: None.

Comments: Bertoni (1914) lists Lysiurus unicinctus and Bertoni (1939) updates the nomenclature to $\mathrm{Ca}$. unicinctus. However, numerous early authors applied the name Ca. unicinctus to the large species now known as Ca. tatouay, or to composite samples containing large and small specimens (Wetzel, 1980). Bertoni's use of the common name Tatú-aí for the species is the same as that used by Azara (1801) and referable to Ca.tatouay.

A review of specimens, localities, and data available for this species in Paraguay was provided by Smith et al. (2011), but a previous record of a specimen (MNHG 1636.032) collected on 12 October 1979 and published in Roguin (1986) was overlooked. The measurements provided confirm the identification and thus the latter is the first record of the species in Paraguay and not Smith et al. (2011).

A reference to two "rather small" and "probably immature" Ca. tatouay seen in Cerrado vegetation at RNBM-Aguara $\tilde{N u}$, Canindeyú department by Lowen et al. (1996) would seem more likely to be this species. The species has not yet been confirmed to occur in Canindeyú, but it may be expected to do so in areas of Cerrado.

Smales (2007) lists Tolypeutes sp. for "the road to Colonia Sergento [sic] E. Lopez, $20 \mathrm{~km}$ north to Estancia Laguna Negra in Caazepa [sic] Province". The specimen he refers to is MNHG 1875.003, a Ca. unicinctus collected by "C. Dlouhy, V. Mahnert \&
C. Vaucher" on an unspecified date at this locality, which is actually in Concepción department.

All Paraguayan specimens are from the Cerrado zone of eastern Paraguay.

Specimens examined: Amambay: "Parque Nacional Cerro Corá (approx. $1.5 \mathrm{~km}$ east of administration office)" MNHNP 919; San Pedro: "Estancia Las Mañanitas" CZPLT 002; "Reserva Natural Laguna Blanca" CZPLT 001.

Specimens not examined: Amambay: "Apa-mi, 10 km S Bella Vista" MNHG 1636,032 (Roguin, 1986); Concepción: "road to Sargento E López, $20 \mathrm{~km}$ north to Estancia Laguna Negra" MNHG 1875.003 (Smales, 2007).

Photographic documentation: Amambay: "Estancia Pa'i Kuará" FPMAM83-89 (Hugo del Castillo in FAUNA Paraguay, 2018).

\section{ChLAMYPHORIDAE: ChLAMyPhORINAE: Fairy Armadillos}

Chacoan Fairy Armadillo

Calyptophractus retusus

(Burmeister, 1863) (FIG. 10)

Chlamyphorus retusus Burmeister 1863:167. Type locality "Santa Cruz de la Sierra" Santa Cruz, Bolivia.

Burmeisteria retusa Myers \& Wetzel (1979: distribution); Seelwische (1980: ethnography).

Chlamyphorus retusus Wetzel (1985a: distribution, taxonomy); Gamarra de Fox \& Martin (1996: records); Gamarra de Fox et al. (1998:

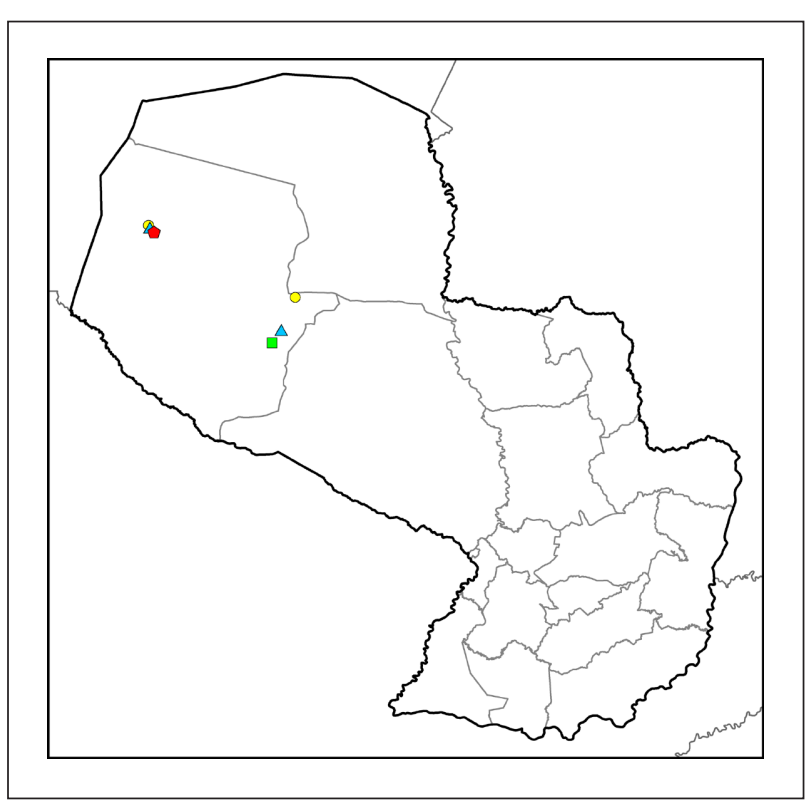

FIGURE 10. Distribution of Calyptophractus retusus in Paraguay. 


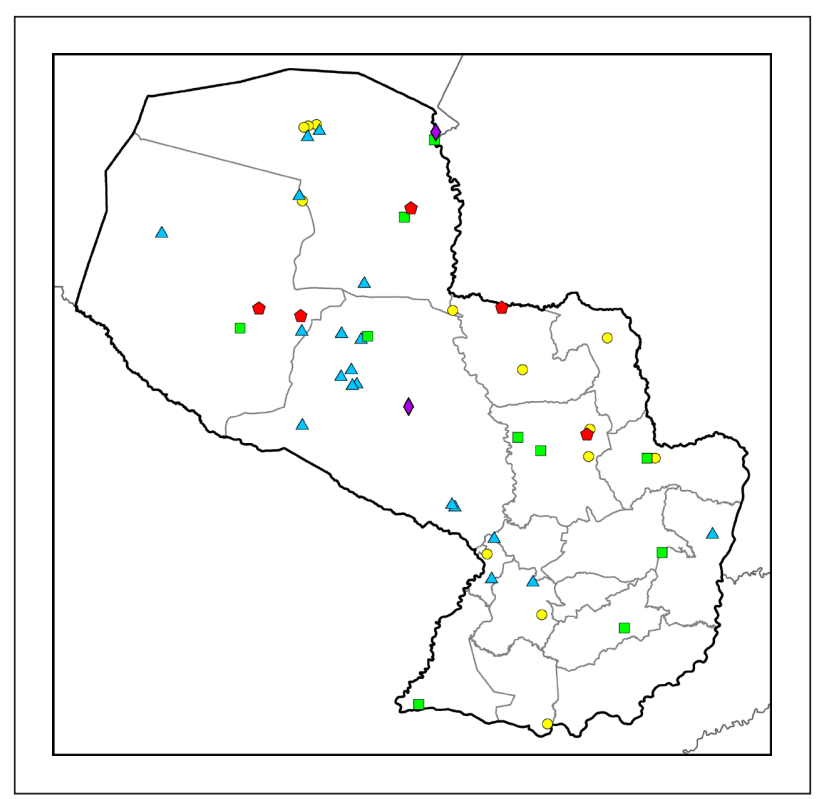

FIGURE 11. Distribution of Euphractus sexcinctus in Paraguay.

conservation); Yahnke et al. (1998: distribution); Neris et al. (2002: distribution); Neris \& Franco Rivarola (2005: guide); Meritt (2008: ecology); Vinke \& Vinke (2014: ecology).

Calyptophractus retusus Abba \& Vizcaíno (2008: specimens); Smith (2008a: ecology); Wetzel et al. (2008: distribution, taxonomy); Ramírez Pinto \& Velázquez (2010: distribution); Smith (2012: conservation); GAT \& OPIT (2014: ethnography); Smith \& Owen (2017: ecology).

Local names: Ayoreo: Ogode (GAT \& OPIT, 2014); Guaraní: Tatu de abrigo (Gamarra de Fox et al., 1998); Nivaclé: Tacluuc (Seelwische, 1980); Spanish: Pichi ciego grande, Tatú de abrigo (Gamarra de Fox \& Martin, 1996); Pichiciego grande (Gamarra de Fox et al., 1998); Pichiciego chaqueño (Morales, 2007); Tatú ciego (Ramírez Pinto \& Velázquez, 2010).

Comments: The species was first officially reported in Paraguay by Myers \& Wetzel (1979) though a photograph dated to 1948 but lacking any data shows the Mennonite collector Jakob Unger with a live specimen in his hand (Vinke \& Vinke, 2014). Yepes (1939) had earlier speculated that the species would likely be present in the Paraguayan Chaco. The skull of this species is illustrated and described for the first time from a Paraguayan specimen in Smith \& Owen (2017).

The species is confined to the Dry Chaco.

Specimens examined: Alcohol specimen lacking data MJUF; Boquerón: "Comunidad Indígena Campo Loro" MNHNP 3362; "Parque Nacional Teniente Enciso" MNHNP 1656; alcohol specimen lacking data at park headquarters.
Specimens not examined: Boquerón: "15 km S Filadelfia" CONN 16881 (Myers \& Wetzel, 1979; Wetzel, 1985a); "Teniente Enciso" CONN 17609 (Myers \& Wetzel, 1979; Wetzel et al., 2008).

Literature citations: Boquerón: "Neu Halbstat" (Vinke \& Vinke, 2014).

Photographic documentation: Boquerón: "Parque Nacional Teniente Enciso" FPMAM67PH (Enrique Bragayrac in FAUNA Paraguay, 2018), probably the same specimen that is located at the PN Teniente Enciso headquarters.

\section{CHLAMYPHORIDAE: EUPHRACTINAE: Hairy Armadillos}

SiX-BANDED ARMADILLO

Euphractus sexcinctus

(Linnaeus, 1758) (FIG. 11)

Dasypus sexcinctus Linnaeus 1758:51. Type locality "America Meridionali" restricted to Pará, Brazil by O. Thomas (1907).

Dasypus sexcinctus Rengger (1830: ecology); Bertoni (1914: list).

Euphractus sexcinctus gilvipes Bertoni (1939: list); Gatti (1985: dictionary).

Euphractus sexcinctus flavimanus Cabrera (1958: taxonomy); Wetzel \& Lovett (1974: specimens).

Euphractus sexcinctus Schmidt \& Martin (1978: parasitology); Myers \& Wetzel (1979: mention); Masi Pallarés \& Benítez Usher (1982: parasitology); Redford (1985: diet); Fujita et al. (1994: parasitology); Brooks (1995: distribution); Fujita et al. (1995: parasitology); Gamarra de Fox \& Martin (1996: records); Gamarra de Fox et al. (1998: conservation); Yahnke et al. (1998: distribution); Villalba \& Yanosky (2000: tracks); Areskoug (2001: habitat); Esquivel (2001: guide); Neris \& Colman (2001: guide); Neris et al. (2002: distribution); Fariña \& Hostettler (2003: distribution); Fast Schartner (2004: guide); Neris \& Franco Rivarola (2005: guide); Yeo et al. (2005: parasitology); Cartes (2007: use); Smith (2007d: ecology); Meritt (2008: ecology); Ramírez Pinto \& Velázquez (2010, distribution); Masi Pallarés (2011: ecology); Smith (2012: conservation); Acosta \& López (2013: parasitology); Velázquez \& Ramírez Pinto (2014: guide).

Euphractus sexinctus Seelwische (1980: ethnography).

Euphractus sexcintus Servicio Forestal Nacional (1982: guide); Hill et al. (2003: use); Abba \& Vizcaíno (2008: specimens).

Euphractes sexcintus Lowen et al. (1996: distribution). Euphractes sexcinctus Nava et al. (2007: parasitology). 
Local names: Aché: Kry'y pura (Esquivel, 2001); Ayoreo: Gatodejai (Neris \& Colman, 2001); Enhlet: Jatjapa (Fast Schartner, 2004); Guaraní: Tatùpoyù (Dobrizhoffer, 1784); Tatú poyú (Azara, 1802); Tatu-poyu (Rengger, 1830); Tatú-podyú, Tatú-vai (Bertoni, 1914); Tatu poju (Servicio Forestal Nacional, 1982); Tatú-pĩtá (Gatti, 1985); Mennonite dialect: Jaelit Jirtillschwien (Fast Schartner, 2004); Nivaclé: Acôtsejaj (Seelwische, 1980); Spanish: Armadillo (Servicio Forestal Nacional, 1982); Tatú-bayo (Gatti, 1985); Armadillo seis bandas (Gamarra de Fox \& Martin, 1996); Armadillo de seis bandas (Neris \& Colman, 2001); Tatú peludo, Peludo grande (Velázquez \& Ramírez Pinto, 2014).

Comments: The use of the Guaraní name Tatùpoyù by Dobrizhoffer (1784) makes it reasonable to suggest that he encountered the species in Paraguayan territory. Azara (1801, 1802) used the same common name, clarifying that it means "armadillo with yellow hands". The common name Tatú-vai listed by Bertoni (1914) approximates to "disgusting armadillo", and is linked to beliefs in certain areas that the animal is inedible because of its habit of eating carrion. Indeed, Dobrizhoffer (1784) described the species as "loathed" because of its tendency to feed on carcasses of mules and horses, and Azara $(1801,1802)$ stated that nobody eats it because of the "foul tasting and smelling meat". Dobrizhoffer (1784) also observed that "lower orders of Spaniards" used the carapace as a plate. The species is widespread in all ecoregions in Paraguay.

MACN 45.028 has locality "San Pedro, Pto. Casado". It is unclear whether this locality refers to Puerto Casado, Alto Paraguay department or to an unknown locality in San Pedro department. The specimen was collected by Cranwell and Giai in December 1944.

Two stuffed specimens on display at the Museo de la Entidad Binacional Yacyretá, Ayolas, Misiones department lack collection data but may have been collected locally, as the species does occur in the area.

Specimens examined: Alcohol specimen lacking data in MNHNP with field number SF05; Mounted specimen lacking data MJUF; "Chaco" SCH 1263; "Paraguay" MACN 11.24; Alto Paraguay: "12 km E of Aguadulce sobre línea 1" MNHNP 519; "Parque Nacional Defensores del Chaco a $8 \mathrm{~km}$ E de la Administración sobre Linea 1" MNHNP 518; "Parque Nacional Defensores del Chaco" MNHNP 515; "San Pedro, Pto. Casado" MACN 45.028; Amambay: "road to monument $1.5 \mathrm{~km} \mathrm{~N}$ casa de Admin. Parque Nacional Cerro Corá" MNHNP 533; Boquerón: "Agua Lidia" MNHNP 517; Canindeyú: "Mbaracayú Wildlife Reserve airstrip at central camp $\left(27^{\circ} 07^{\prime} \mathrm{S}, 57^{\circ} 55^{\prime} \mathrm{W}\right)^{\prime \prime}$ MNHNP 918; Central: "San Lorenzo" SCH 222; Concepción: "Paso Barreto" uncatalogued specimen in MNHNP with field number SFUH40; Itapúa: "Isla Yacyreta" MNHNP 1077; Paraguarí: "Parque Nacional Ybycuí muerto en el camino a $1 \mathrm{~km} \mathrm{~N}$ de la Fundación La Rosada" MNHNP 520; San Pedro: "Reserva Natural Laguna Blanca" CZPLT 420, 425.

Specimens not examined: "Paraguay" NHM 1902.4. 7.45, MHNG 1968, 092, MZB 82-7425; Alto Paraná: "Refugio Biológico Itabó, Itaipú" CBMI 0110; Alto Paraguay: "60 km by road N Fortín Madrejón" UMMZ 125579; "114 km W Puerto Sastre" CONN 19512; "Aguadulce, 90 km NE Madrejón" CONN 19935; "north of Filadelfia" TTU 79880; Boquerón: "km 654.5 Transchaco road" CONN 17502; "Sargento Rodríguez, km 764 Transchaco road" CONN 17501, 17607; "surroundings of $22^{\circ} 34^{\prime} \mathrm{S}, 59^{\circ} 20^{\prime} \mathrm{W}^{\prime \prime}$ MTD 24889; "road to Loma Plata $4 \mathrm{~km} \mathrm{~N}$ Transchaco" CONN 20870; Central: "Colonia Nueva Italia" FMNH 54325; "Salado River" USNM 1482; Paraguarí: "Sapucay" NHM 1902.4.7.45; Presidente Hayes: " $4 \mathrm{~km}$ E of Transchaco bridge, right bank of Rio Verde, Juan de Zalazar" CONN 15964 (Wetzel \& Lovett, 1974); "4 km NW of Line Camp, Juan de Zalazar" CONN 16646; "8 km NE Juán de Zalazar" UMMZ 134014; "Laguna Pora, 85 km E Loma Plata" CONN 19958, 19960, 20030, 20352, 20432; " km 94 Transchaco road" CONN 20869; "km 100 approximately Transchaco road" CONN 10870; "295 km (by road) Villa Hayes" MVZ 145359, 145360; "Estancia Elsita" SMNH 593524, 593525; "Juan de Zalazar" CONN 15963 (Wetzel \& Lovett, 1974).

Literature citations: Alto Paraguay: "Puerto Rio Negro" (Masi Pallarés \& Benítez Usher, 1982); Alto Paraná: no specific locality (Bertoni, 1939); "Estancia San Antonio" (Lowen et al., 1996); Boquerón: "Gran Siete" (Areskoug, 2001); "surroundings of $20^{\circ} 46-57^{\prime} \mathrm{S}, 5^{\circ} 48-53^{\prime} \mathrm{W}^{\prime \prime}$ (Ziegler et al., 2002); Caazapá: "Reserva Natural Tapytá" (Velázquez \& Ramírez Pinto, 2014); Canindeyú: "Mbaracayú Reserve" (Hill et al., 2003); Presidente Hayes: "General Díaz" (Masi Pallarés \& Benítez Usher, 1982); "Laguna Pora" (Redford, 1985); San Pedro: "Chamorro Cué outskirts of San Pedro de Ycuamandyyú" (Fujita et al., 1995); "Pirí Pucú outskirts of San Pedro de Ycuamandyyứ" (Fujita et al., 1995).

Photographic documentation: Alto Paraguay: "Toro Pampa" FPMAM1003-1004 (Paul Smith in FAUNA Paraguay, 2018); Boquerón: "Central Chaco" FPMAM46 (Paul Smith in FAUNA Paraguay, 2018); "near Loma Plata" FPMAM924 (Paul Smith in FAUNA Paraguay, 2018); Concepción: "Cercanías de San Carlos del Apa" FPMAM1186PH (Sergio D. Ríos in FAUNA Paraguay, 2018); San Pedro: "Reserva Natural Laguna Blanca" FPMAM45, 47 (Paul Smith in FAUNA Paraguay, 2018).

Reliable observations: Alto Paraguay: "Tres Gigantes" (Paul Smith 4 September 2018). 


\section{GREATER HAIRY ARMADILlO \\ Chaetophractus villosus \\ (Desmarest, 1804) (FıG. 12)}

lor[icatus]. villosus Desmarest 1804:28. Based on Azara (1801). Type locality "Les Pampas", Buenos Aires, Argentina.

Chaetophractus sp. Wetzel \& Lovett (1974: specimens); Areskoug (2001: habitat).

Chaetophractus villosus Myers \& Wetzel (1979: distribution); Gamarra de Fox \& Martin (1996: records); Gamarra de Fox et al. (1998: conservation); Yahnke et al. (1998: distribution); Neris et al. (2002: distribution); Neris \& Franco Rivarola (2005: guide); Nava et al. (2007: parasitology); Abba \& Vizcaíno (2008: specimens); Meritt (2008: ecology); Smith (2008b: ecology); Smith (2012: conservation).

Choetophractus villosus Seelwische (1980: ethnography).

Chaethophractus villosus Ramírez Pinto \& Velázquez (2010: distribution).

Local names: Guaraní: Tatú-velu (Wetzel \& Lovett, 1974); Poju'i (Neris et al., 2002); Nivaclé: Casuts'i (Seelwische, 1980); Spanish: Peludo (Azara, 1802); Peludo argentino, Tatú peludo (Gamarra de Fox \& Martin, 1996); Pichi peludo, Quirquincho grande (Neris et al., 2002).

Comments: Azara (1802) stated that the species did not occur in Paraguay. The species was first cited for Paraguay by Wetzel (1977) and the first specimens were reported by Myers \& Wetzel (1979). Unpublished specimens SMNH 593510 (collected

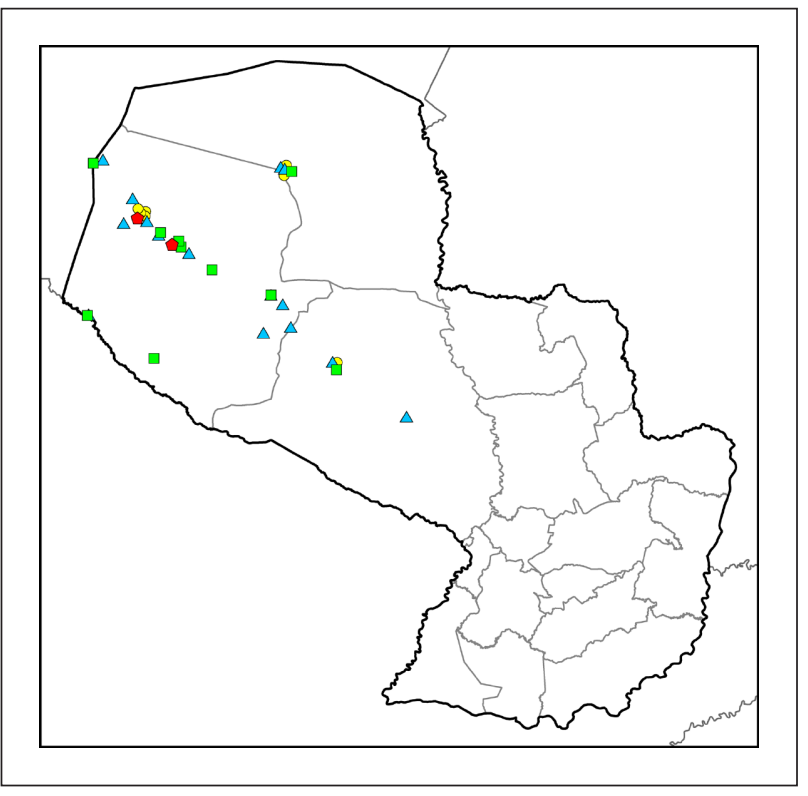

FIGURE 12. Distribution of Chaetophractus villosus in Paraguay. by Olrog in January 1948), FMNH 54352 (collected by Willim 6 September 1945), and FMNH 63865 (collected by Unger 18 December 1945) all predate the published specimens. The species is commonest in the Dry Chaco, with marginal occurrence in the Humid Chaco and possibly the Cerrados del Chaco ecoregions.

Specimens examined: Alto Paraguay: " $5 \mathrm{~km}$ north Madrejón admin., PN Defensores del Chaco" MNHNP 530; Boquerón: "5 km south Madrejón admin., PN Defensores del Chaco" MNHNP 524; "10 km al sur de Madrejón por el camino" MNHNP 529, 531; "PN Tte Enciso approx $3 \mathrm{~km}$ este sobre el límite sur" MNHNP 522; "PN Tte Enciso 500 km SE de la casa de control por la picada" MNHNP 532; "Ruta Transchaco $3 \mathrm{~km} \mathrm{~N}$ casa de admin, PN Tte Enciso" MNHNP 525; "Ruta Transchaco 10 km N casa de admin, PN Tte Enciso" MNHNP 527; Presidente Hayes: "Salazar, Galpón" uncatalogued alcohol specimen in MNHNP with field number SF40.

Specimens not examined: Alto Paraguay: "6 km NW of Madrejón on old road to Cerro León" FLMNH 20651; "Fortín Madrejón" CONN20435; Boquerón: "25 km W Teniente Enciso" CONN 17500; "km 655 Teniente Enciso" CONN 18064; "Copagro, km 590 Transchaco" CONN 18308, 18350, 18952 (Wetzel \& Lovett, 1974); "390 km NW (by road) Villa Hayes" MVZ 145361; "Colonia Neuland" MNHNP 1155; "Dr. Pedro P. Peña" CONN 19925; "Estancia Iparoma, 19 km N Filadelfia" CONN 19961; "Garrapatal" CONN 18044; "Guachalla, Rio Pilcomayo, 580 km W Asunción" FMNH 54352; "Orloff" FMNH 63865; "Sargento Rodríguez, Transchaco km 764" CONN 17606; Presidente Hayes: " 8 km NW headquarters, Estancia Juan de Salazar" MSB 57375; "Estancia Hermosa" SMNH 593510; "Estancia Samaklay” TTU 80408.

Literature citations: Alto Paraguay: "Fortín Madrejón" (Myers \& Wetzel, 1979); Boquerón: "25km by road NW Mariscal Estigarribia" (Myers \& Wetzel, 1979); "27 km by road NW Teniente Ochoa" (Myers \& Wetzel, 1979); "30 km by road NW Teniente Ochoa" (Myers \& Wetzel, 1979); "35 km by road SE Teniente Enciso" (Myers \& Wetzel, 1979); "42 km by road SE Teniente Pratt Gill airforce base" (Myers \& Wetzel, 1979); "Dr. Pedro P. Peña" (Myers \& Wetzel, 1979); "Estancia Iparoma, $19 \mathrm{~km}$ by road N Filadelfia" (Myers \& Wetzel, 1979); "Fortín Sargento Rodríguez" (Myers \& Wetzel, 1979); Presidente Hayes: "Juan de Salazar" (Myers \& Wetzel, 1979).

Photographic documentation: Boquerón: "Parque Nacional Teniente Enciso" FPMAM59 (Silvia Centrón in FAUNA Paraguay, 2018); "Ruta Transchaco km 634" FPMAM60-66 (Paul Smith in FAUNA Paraguay, 2018). 


\section{SCREaming Hairy ARMadillo \\ Chaetophractus vellerosus \\ (Gray, 1865) (FIG. 13)}

Dasypus vellerosus Gray 1865:376. Type locality "Santa Cruz de la Sierra", Santa Cruz, Bolivia.

Chaetophractus vellerosus Myers \& Wetzel (1979: distribution); Seelwische (1980: ethnography); Wetzel (1985b: distribution); Gamarra de Fox \& Martin (1996: records); Gamarra de Fox et al. (1998: conservation); Yahnke et al. (1998: distribution); Neris et al. (2002: distribution); Neris \& Franco Rivarola (2005: guide); Abba \& Vizcaíno (2008: specimens); Meritt (2008: ecology); Smith (2008d: ecology); Smith (2012: conservation).

Local names: Guaraní: Tatu peludo (Gamarra de Fox \& Martin, 1996); Tatu Poju'i (Neris et al., 2002); Nivaclé: Yiclôôj (Seelwische, 1980); Spanish: Piche llorón (Gamarra de Fox \& Martin, 1996); Pichi llorón (Gamarra de Fox et al., 1998); Armadillo, Pichi peludo (Neris et al., 2002).

Comments: The species was first reported for Paraguay by Myers \& Wetzel (1979). The first specimen was CONN 16895 collected by R. L. Martin on 19 July 1974.

MACN 47.378 listed with locality "Alto Paraná, Capitán Meza" is a specimen collected by Adolfo Neuntenfel and purchased by the museum on 30 October 1947. Capitán Meza is in Itapúa department, not Alto Paraná and may have been a shipping locality as MACN 47.377 (Ca.tatouay) has the same details. Chaetophractus vellerosus does not occur east of the Paraguay River, so this locality is undoubtedly an error. The species is known only from the Dry Chaco ecoregion with marginal occurrence in the Humid Chaco.

Specimens examined: Alto Paraguay: "Estancia Campo Grande, $1^{\circ} 46.511$ 'S, 59 $46.515^{\prime} \mathrm{W}^{\prime \prime}$ MNHNP 3364; Boquerón: " $3 \mathrm{~km}$ norte Filadelfia sobre el camino para Montanía" MNHNP 521; "60 km E Filadelfia" MNHNP 523; "PN Tte Enciso" MSB 54419; "Ruta Transchaco km 697" MSB 54075; "Filadelfia" MNHNP 526, 528, MSB 58990; “Transchaco camino a PN Médanos del Chaco" a carapace lacking data at park headquarters, but deposited there by Hugo del Castillo 12 August 2010 (HDC pers. comm.); Itapúa: "Alto Paraná, Capitán Meza" MACN 47.378.

Specimens not examined: "Paraguay" FMNH 157156; Boquerón: "Estancia Iparoma, 19 km N Filadelfia" CONN 19927, 19928, 19929, 20015; Presidente Hayes: " $30 \mathrm{~km} \mathrm{~W}$ of guesthouse, km 320 Transchaco, Juan de Zalazar" CONN 16895.

Literature citations: Alto Paraguay: "Parque Nacional Defensores del Chaco" (Yahnke et al., 1998); Boquerón: "24 km by road NW Teniente Ochoa" (Myers \& Wetzel, 1979); "27 km by road NW

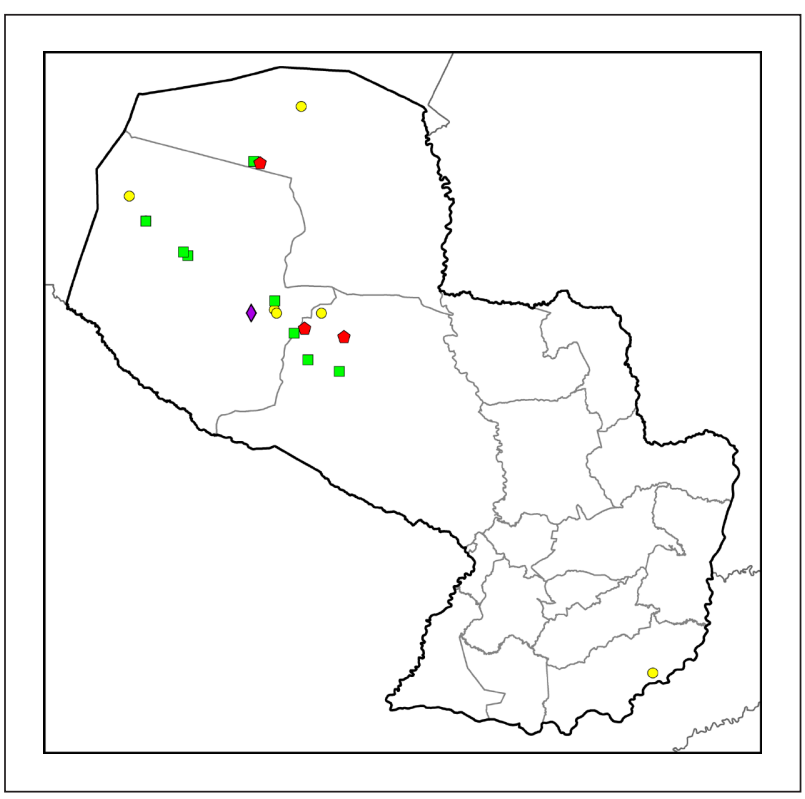

FIGURE 13. Distribution of Chaetophractus vellerosus in Paraguay.

Teniente Ochoa" (Myers \& Wetzel, 1979); "390 km by road (Route 9) NW Villa Hayes" (Myers \& Wetzel, 1979); "Estancia Iparoma, $19 \mathrm{~km}$ by road N Filadelfia” (Myers \& Wetzel, 1979); "Parque Nacional Teniente Enciso" (Yahnke et al., 1998); Presidente Hayes: "between Para Todos and 25 Leguas" (Myers \& Wetzel, 1979); "Juan de Zalazar" (Myers \& Wetzel, 1979; Wetzel 1985b).

Photographic documentation: Alto Paraguay: "Parque Nacional Defensores del Chaco" FPMAM53 (Sjeff Ollers in FAUNA Paraguay, 2018); Presidente Hayes: "Cuenca Upper Yacaré Sur" FPMAM54-56 (Paul Smith in FAUNA Paraguay, 2018); "Laguna Capitán" FPMAM48-52 (Paul Smith in FAUNA Paraguay, 2018).

Reliable observations: Boquerón: "Fortín Toledo" pair in coitus 23 September 2014 (Paul Smith).

\section{MYRMECOPHAGIDAE: Anteaters}

\section{Giant ANTEATER}

Myrmecophaga tridactyla

Linnaeus, 1758 (FIG. 14)

[Myrmecophaga] tridactyla Linnaeus 1758:35. Type locality "America Meridionali", restricted to Pernambuco, Brazil by O. Thomas (1911).

Myrmecophaga jubata Rengger (1830: ecology); Bertoni (1914: list); Seelwische (1980: ethnography).

Myrmecophaga tridactyla Bertoni (1939: list); Seelwische (1980: ethnography); Masi Pallarés \& Benítez Usher (1982: parasitology); Gatti (1985: dictionary); Brooks (1995: distribution); Gamarra de Fox \& Martin (1996: records); Lowen et al. (1996: distribution); Gamarra de Fox et al. (1998: conservation); Villalba \& 
Yanosky (2000: tracks); Esquivel (2001: guide); Neris et al. (2002: distribution); Fast Schartner (2004: guide); Neris \& Franco Rivarola (2005: guide); Cartes (2007: use); Nava et al. (2007: parasitology); Smith (2007a: ecology); Abba \& Vizcaíno (2008: specimens); Meritt (2008: ecology); Itaipú Binacional (2010: guide); Ramírez Pinto \& Velázquez (2010: distribution); Smith (2012: conservation); Velázquez \& Ramírez Pinto (2014: guide); Epp (2018: guide).

Myrmecophaga tridactyla tridactyla Wetzel \& Lovett (1974: specimens).

Myrmechophaga tridactyla Yahnke et al. (1998: distribution).

Mymecophaga tridactyla Fariña \& Hostettler (2003: distribution).

Local names: Aché: Kuare (Fariña \& Hostettler, 2003); Enhlet: Naayem' (Unruh \& Kalisch, 1997); Anim (Fast Schartner, 2004); Guaraní: Tamanduà, Yoquì, Nurumì (Dobrizhoffer, 1784); Nurumi, Yoquí (Azara, 1802); Yurumi (Rengger, 1830); Djurumí (Bertoni, 1914); Tamanduá guasú, Yurumí (Bertoni, 1939); Yurú Mí (Wetzel \& Lovett, 1974); Yokĩ, Cúmbirí (Gatti, 1985); Juru mi (Fariña \& Hostettler, 2003); Jurumi (Neris et al., 2002); Mbyá: Kaguare guachu (Cadogan, 1992); Nivaclé: S'uclaj (Seelwische, 1980); Mennonite dialect: Emstjiboa (Fast Schartner, 2004); Spanish: Osso hormigero (Dobrizhoffer, 1784); Oso hormiguero (Azara, 1802); Oso hormiguero grande, Tamanduá bandera (Velázquez \& Ramírez Pinto, 2014).

Comments: The species was first mentioned for Paraguay by Dobrizhoffer (1784) as Ursus Formicarius. Azara (1801, 1802) stated that the common name Nurumi or Yurumí means "small mouth".

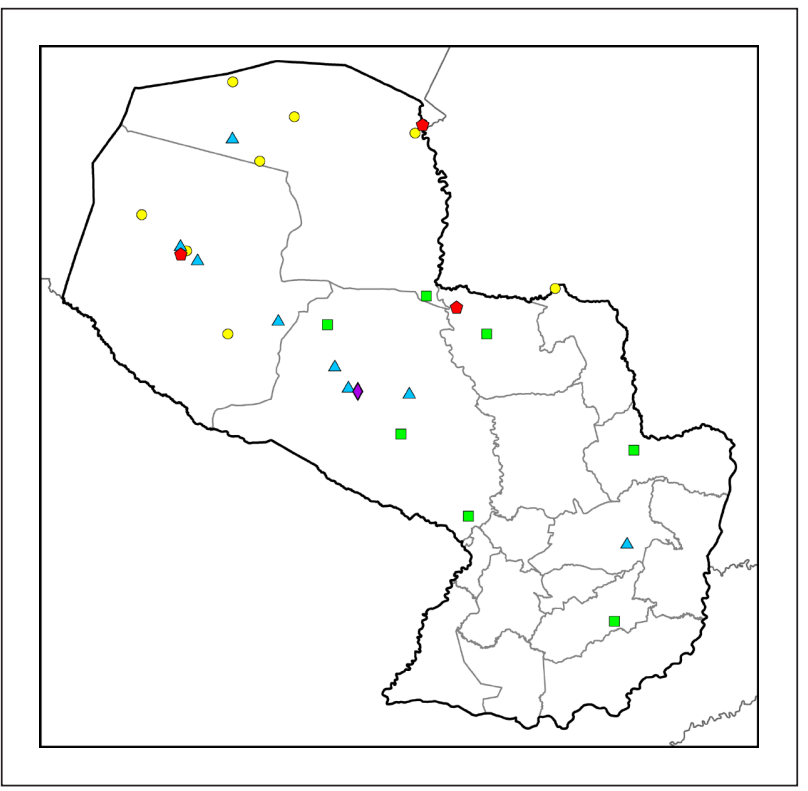

FIGURE 14. Distribution of Myrmecophaga tridactyla in Paraguay.
It occurs (or did occur) in all ecoregions, but is most common west of the Paraguay river especially in the Pantanal and Humid Chaco ecoregions. In the Oriental region it is now encountered regularly only in the Cerrado ecoregion, and greatly reduced in the Atlantic Forest zone.

A stuffed specimen on display at the Museo de la Entidad Binacional Yacyretá, Ayolas, Misiones department lacks collection data. Several specimens of this species are also exhibited at the Museo Schade, one of which, a juvenile, lacks a specimen number or collection data and thus is not listed below.

Specimens examined: Mounted specimens of adult and juvenile lacking data MJUF; "Chaco" $\mathrm{SCH}$ 384, 385, 1249, 1250; Alto Paraguay: "20¹1.18'S, $58^{\circ} 15.36^{\prime} \mathrm{W}^{\prime \prime}$ MNHNP 3361; "35 km west of administration PN Defensores del Chaco" (= $35 \mathrm{~km}$ west of Madrejón?) MNHNP 1124; "Aguadulce" MNHNP 1125; "Palmar de las Islas" MNHNP 3255; "Tres Gigantes" a locally collected skull lacking specimen details in onsite museum; Amambay: "Bella Vista" MNHNP 1189; Boquerón: no specific locality MNHNP 1169; "km 595 Ruta Trans Chaco" MNHNP 1185; "Estancia San Ramón 48 km west of Colonia Neuland" MNHNP 1123; "runway Teniente Enciso" MNHNP 818.

Specimens not examined: "Paraguay" CONN 24976, MACN 23.28, 27.26, 29.238, 29.243, 30.242 (Abba \& Vizcaíno, 2008); "Paraguay, Zoo Buenos Aires" MACN 3.59, 30.35, 25.190 (Abba \& Vizcaíno, 2008); Alto Paraguay: "77 km NW of Madrejon, 15 km N on Linea 6" CONN 19524; Boquerón: no specific locality AMNH-M-48187; “410 km NW (by road) Villa Hayes" MVZ 145368; "Copagro, 4 km SE Transchaco km 589" CONN 18851; Caaguazú: "junction of Iguazú and Yuqueri rivers" MCZ 28622, 30738; Presidente Hayes: "approx 275 km NW by rd NW Villa Hayes" UMMZ 156446; "Est. Juan de Zalazar" CONN 16067 (Wetzel \& Lovett, 1974); "Waikthlatingmayalwa" UMZC E562, E563.

Literature citations: Boquerón: "surroundings of $22^{\circ} 34^{\prime} \mathrm{S}, 5^{\circ} 9^{\circ} 0^{\prime} \mathrm{W}^{\prime \prime}$ (Zeigler et al., 2002); Canindeyú: "Reserva Bosque Mbaracayú" (Lowen et al., 1996; Esquivel, 2001; Fariña \& Hostettler, 2003); Caazapá: "Reserva Natural Tapytá" (Velázquez \& Ramírez Pinto, 2014); Concepción: "Garay Cué" (Masi Pallarés \& Benítez Usher, 1982); Presidente Hayes: "km 200-250 of Ruta Transchaco" (Meritt, 2008).

Photographic documentation: Alto Paraguay: "Tres Gigantes" FPMAM1019-1025, 1075-1077 (Paul Smith in FAUNA Paraguay, 2018); Boquerón: "Ruta Transchaco km 600" FPMAM34-36 (Paul Smith in FAUNA Paraguay, 2018); Concepción: "Ruta Concepción-Vallemí, 30 km de Vallemí" FPMAM1188PH (Sergio D. Ríos in FAUNA Paraguay, 2018)". 
Reliable observations: Presidente Hayes: "km 291 Ruta Trans Chaco" (Paul Smith 1 September 2018).

\section{SOUTHERN TAMANDUA}

Tamandua tetradactyla

(Linnaeus, 1758) (FIG. 15)

Myrmecophaga tetradactyla Linnaeus 1758:35. Type locality "America meridionali". Restricted to Pernambuco, Brazil by O. Thomas (1911).

Myrmecophaga tetradactyla Rengger (1830: ecology).

Tamandua tetradactyla Quijada (1910: specimen); Cabrera (1912: specimen); Bertoni (1914: list); Bertoni (1939: list); Wetzel \& Lovett (1974: specimens); Seelwische (1980: ethnography); Masi Pallarés \& Benítez Usher (1982: parasitology); Gatti (1985: dictionary); Roguin (1986: distribution); Brooks (1995: distribution); Gamarra de Fox \& Martin (1996: records); Lowen et al. (1996: distribution); Gamarra de Fox et al. (1998: conservation); Yahnke et al. (1998: distribution); Villalba \& Yanosky (2000: tracks); Esquivel (2001: guide); Neris et al. (2002: distribution); Fariña \& Hostettler (2003: distribution); Hill et al. (2003: use); Neris \& Franco Rivarola (2005: guide); Cartes (2007: use); Nava et al. (2007: parasitology); Smith (2007e: ecology); Abba \& Vizcaíno (2008: specimens); Meritt (2008: ecology); Vinke \& Vinke (2008: popular); Smith (2012: conservation); Velázquez \& Ramírez Pinto (2014: guide); Epp (2018: guide).

Local names: Aché: Kuaremini (Esquivel, 2001); Guaraní: Cagüaré (Azara, 1802); Caguaré (Rengger, 1830); Kaaguaré (Bertoni, 1914); Kaguaré (Bertoni, 1939); Kaguare (Neris et al., 2002); Tamandua (Fariña \& Hostettler, 2003); Tamanduá (Vinke \& Vinke, 2008); Tamandua miri (Ramírez Pinto \& Velázquez, 2010); Mbyá: Kaguare mirĩ (Cadogan, 1992); Nivaclé: S'uclataj (Seelwische, 1980); Spanish: Oso hormiguero chico (Azara, 1802); Oso mielero (Gamarra de Fox \& Martin, 1996); Oso melero (Esquivel, 2001).

Comments: The species was first cited for Paraguay by Azara $(1801,1802)$ who states that the common name Cagüaré is a shortened form of Caaigüaré, roughly meaning "smelly beast of the forest". Cabrera (1912) mentions a melanistic specimen with locality "Paraguay" in the Museo de Ciencias Naturales de Madrid (MCNM 490).

Occurs in all ecoregions, though it reaches its greatest abundance in the Humid Chaco, Cerrado and Cerrados del Chaco ecoregions.

Specimens examined: Two mounted specimens lacking data MJUF; "Chaco" SCH 165, 680; "Paraguay" MACN 6.22, 11.38, 17.102, 17.110, 21.25, 30.202 (Abba \& Vizcaíno, 2008), MCZ 28640,

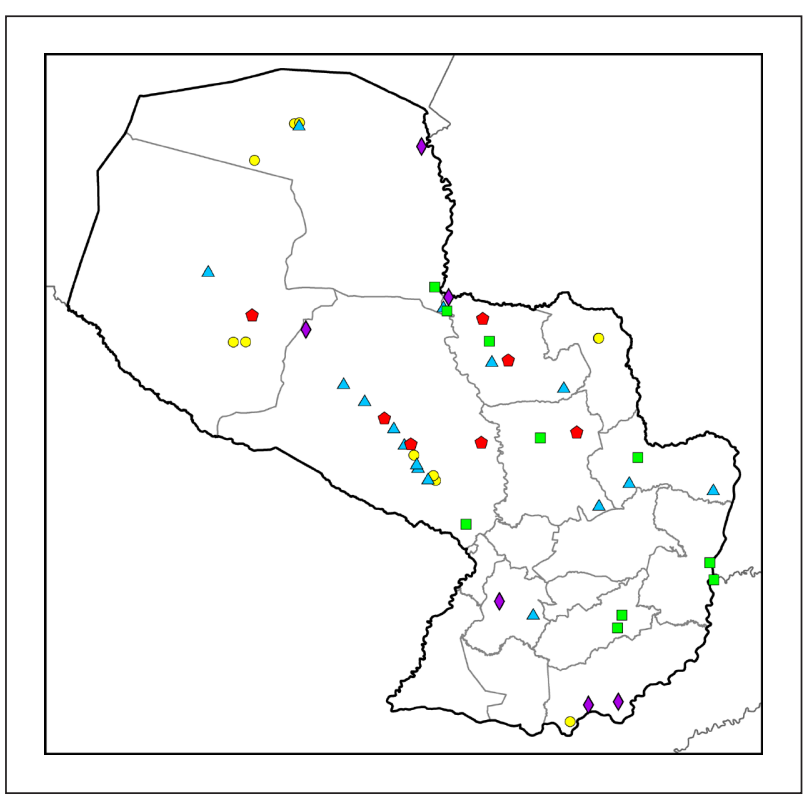

FIGURE 15. Distribution of Tamandua tetradactyla in Paraguay.

MCNM 490; "Paraguay, Zoo Buenos Aires" MACN 4.391, 17.104, 24.164 (Abba \& Vizcaíno, 2008); Alto Paraguay: "PN Defensores del Chaco $3.5 \mathrm{~km}$ west of Aguadulce" MNHNP 508; "4 km west of Aguadulce" MNHNP 507; "PN Defensores del Chaco, Cerro León" MNHNP 1068; Amambay: "PN Cerro Corá" MNHNP 1070; "PN Cerro Corá 1 km west of administration" MNHNP 509; Boquerón: "Estancia La Gama" MNHNP 1071, 1072, 1073; "Estancia San Ramón 48 km west of Colonia Neuland"; Itapúa: "Estancia Melgarejo, Isla Yacyreta" MNHNP 1117; Presidente Hayes: "km 137 Ruta Trans Chaco" MNHNP 1193; "Approx. km 139 of Ruta Trans Chaco" MNHNP 506; "Ruta Trans Chaco km 182" MNHNP 1074.

Specimens not examined: "Paraguay" CBMI 0259; MNC (Quijada, 1910), MCNM 490 (Cabrera, 1912), MZB 82-7470, MCZ 28640; Alto Paraná: "Vivero Forestal, Itaipú" CBMI 0001; Alto Paraguay: "Aguadulce 123 km E Mayor Pablo Lagarenza" CONN 19821; "Puerto Casado, Estancia Casilda" MACN 45.022 (Abba \& Vizcaíno, 2008); Boquerón: "km 567 Transchaco" CONN 18855; Canindeyú: “6.3 km by road NE Curuguaty" UMMZ 124687, 124688; "Pozuelo" CBMI 0039; Concepción: "30km SE Estancia Santa Maria" MNHG 1689.059 (Roguin, 1986); Paraguarí: "Parque Nacional Ybycuí" UMMZ 146508; Presidente Hayes: " $24^{\circ} 23^{\prime} \mathrm{S}, 58^{\circ} 06^{\prime} \mathrm{W}^{\prime}$ MTD 24888 (Zeigler et al., 2002); "km 165 Ruta Transchaco" TTU 80575; "km 169 Ruta Transchaco" CONN 18294; "km 200 Ruta Transchaco" CONN 17035; "km 285 Ruta Transchaco" CONN 16831; "Chaco Experimental Station 295 km NW (by road) Villa Hayes" MVZ 145369; "km 230 rte Tranchaco" MNHG 1689.060 (Roguin, 1986); San Pedro: “46 km by road ENE Mbutuy" UMMZ 146509. 
Literature citations: Alto Paraná: "Puerto Bertoni" (Bertoni, 1914, 1939); Caazapá: "Parque Nacional Caaguazú" (Lowen et al., 1996); "Reserva Natural Tapytá" (Velázquez \& Ramírez Pinto, 2014); Canindeyú: "Reserva Bosque Mbaracayú" (Lowen et al., 1996; Esquivel 2001; Fariña \& Hostettler, 2003, Hill et al., 2003); Concepción: "Garay Cué" (Masi Pallarés \& Benítez Usher, 1982); Presidente Hayes: "Reserva Natural Privada Golondrina" (Lowen et al., 1996); "Transchaco approximately km 345" (Wetzel \& Lovett, 1974).

Photographic documentation: Concepción: no specific locality FPMAM38-39PH (Hugo del Castillo in FAUNA Paraguay, 2018); "Camino a San Carlos del Apa" FPMAM1189PH (Sergio D. Ríos in FAUNA Paraguay, 2018); Cordillera: "Cabañas" FPMAM1190PH (Joaquín Movia in FAUNA Paraguay, 2018); Presidente Hayes: "Estancia Cachiveo" FPMAM40-41PH (Karen Penayo in FAUNA Paraguay, 2018); "Ruta Transchaco km 200" FPMAM42-43 (Paul Smith in FAUNA Paraguay, 2018); “Ruta Transchaco km 250" FPMAM903-904PH (Paul Smith in FAUNA Paraguay, 2018); San Pedro: "Reserva Natural Laguna Blanca" (PLT camera trap photo 10 May 2012).

Reliable observations: Alto Paraguay: "Tres Gigantes" (Paul Smith 2 September 2018); "Linea 2 6 km south of Bahía Negra" 6 September 2018 (Paul Smith); Boquerón: "Fortín Toledo" 29 July 2015 (Paul Smith); Concepción: "Estancia Tres Hermanas del Apa" 8 August 2011 (Paul Smith); Itapúa: "near Carmen del Paraná" roadkill 10 January 2017 (Paul Smith); "Hotel Tirol" 2007 (Paul Smith); Paraguarí: "near Quiindy" roadkill 10 January 2017 (Paul Smith); Presidente Hayes: "Laguna Capitán" 2 September 2018 (Paul Smith).

\section{Rejected Species}

\section{BRADYPODIDAE: Sloths}

\section{BROWN-THROATED THREE-TOED SLOTH \\ Bradypus variegatus \\ Schinz, 1825}

Brad[ypus]. variegatus Schinz, 1825:510. Type locality "Südamerika," restricted to "Brasilien (wahrscheinlich Bahia) [Brazil, probably Bahia]" by Mertens (1925).

Bradypus tridactylus Bertoni (1914: hypothetical); Bertoni (1939: hypothetical).

Bradypus variegatus Gamarra de Fox \& Martin (1996: no records); Gamarra de Fox et al. (1998: no records); Yahnke et al. (1998: possible); Ziegler et al. (2002: specimen); Morales (2007: list); Abba \& Vizcaíno (2008: specimens); Smith (2012: rejected).
Local names: Guaraní: Ao-aó (Bertoni, 1914); Spanish: Perezoso bayo (Gamarra de Fox et al., 1998); Peresozo [sic] de tres dedos (Morales, 2007).

Comments: Bertoni $(1914,1939)$ lists B. tridactylus as a hypothetical species, stating that it has a distribution that "encircles Paraguay almost completely", but which in Misiones (presumably Argentina, not Paraguay) "does not come close to the banks of the Paraná". In fact the only mention of the species in Misiones, Argentina seems to be Holmberg (1895) who claimed to have seen skins. Superina et al. (2010:124), however, state that it is "historically absent from ... northeastern Argentina" and that the southernmost confirmed record of the species in Brazil is in Londrina, Paraná state where it is now considered extinct.

Bertoni $(1914,1939)$ mentions hearsay reports of the species in the "yerbales del norte" ("yerba mate plantations of the north"). Though it is unclear exactly where this refers to geographically (perhaps the northern Oriental region?), it raises an interesting possibility of a crossover between scientific methodology and local culture. The common name given for the species of Ao-aó, also happens to be the name of a legendary beast in the Guaraní folklore. Regardless of this, the association of the common name of the species with local legend casts serious doubt on the validity of secondhand reports by non-specialists in a scientific context.

A complete skeleton specimen (MACN 4125) dating from 1904 of an individual from Buenos Aires Zoo that supposedly came from "Paraguay" lacks precise locality data and is of dubious provenance (Abba \& Vizcaíno, 2008). A specimen in the Jakob Unger Museum, Filadelfia listed by Ziegler et al. (2002) and prepared by J. Unger is from Bolivia according to the museum records (L. Bergen, pers. comm.).

Gamarra de Fox \& Martin (1996) and Gamarra de Fox et al. (1998) list the species as one with "problems with records" for Paraguay, and Yahnke et al. (1998) list it as "possible" in Defensores del Chaco National Park. Though the species is frequently cited as present in Paraguay, there remains no evidence to support its presence in the country (Smith, 2012).

Specimens examined: Mounted specimen in Filadelfia Museum.

Specimens not examined: MACN 4125 "Paraguay" from Buenos Aires Zoo 1904, complete skeleton (Abba \& Vizcaíno, 2008).

Literature citations: None.

Photographic documentation: None.

Reliable observations: None. 


\section{DIscussion}

Despite being one of the most conspicuous and charismatic mammal groups, the Xenarthra are poorly represented in Paraguayan museum collections and accompanying specimen data are frequently vague or absent. It is lamentable that important specimens are housed in collections that are improperly curated, lacking even basic specimen data, whilst the lack of available funding for the national MNHNP collection is reflected in the deteriorating state of many of the specimens housed there, despite the best efforts of the professional staff to combat it.

Specimen collection localities are biased towards a few, well-sampled sites (particularly in the Central Chaco and Mbaracayú Reserve) whilst other areas of the country have been virtually unsampled (notably the southern Oriental region). Consequently, numbers of specimens represented in collections are heavily biased towards those that are most numerous in the most sampled locations (TABLE 1), thus failing to provide an accurate documentation of the true distribution even of the most numerous species in Paraguay. The current representation of Xenarthra in museum collections is far from adequate and the age of many of the specimens provides only limited and dated information on distribution and status in a country with a rapidly changing landscape.

The availability of reliable and recent information is arguably the most important factor in the accurate determination of the conservation status of a given species, and the partial updating of old information with infrequent and unfocused rapid ecological assessments (REAs) is unsatisfactory and, given the tendency for these to repeat data provided in previous REAs from nearby localities, is often a dangerously misleading substitute. Leaving aside the logistical and financial difficulties of data gathering, we consider it necessary for some kind of estimation of the reliability and modernity of the data upon which conservation assessments are made to accompany such designations in order to correctly highlight weaknesses in the data pool, indicate priorities for investigation, and give weight to real scientific data over opinion and hearsay. A consequence of this has been the repeated appearance in Paraguayan lists of species for which no actual documentation exists, as is the case with B. variegatus. We hope that the information provided here will assist with that objective.

An additional and often ignored effect of the lack of available data in conservation assessments is the unconscious tendency for assessors to give more emphasis to the perceived reduction in records of large, charismatic species such as $P$. maximus and M. tridactyla than to those of less charismatic yet less-frequently observed species.
This bias is reflected in a perceived greater threat status to these "flagship species", coupled with an equally unsupported assumption that the smaller species are less threatened and hence are probably being overlooked. Without real population and distributional data, national threat statuses cannot accurately represent the reality of the conservation statuses of the species they assess, and in fact more accurately reflect the private concerns or level of experience and knowledge of the assessors. The subsequent use of national threat statuses estimated in this way for the assessment of global threat statuses may be having grave consequences for some species that are rapidly, but quietly, declining. Perhaps more worrying is that these weakly supported decisions (by no means unique to xenarthrans) are being relied upon by government officials to determine quotas for the harvesting of wildlife by private, profit-seeking companies. Inaccurate conservation assessments may in fact be exacerbating the problem rather than easing it (Smith, 2012).

This paper represents a first attempt to critically quantify the available data on the distribution of Paraguayan Xenarthra by highlighting gaps in our knowledge, clarifying misunderstandings, and distinguishing between different types of records so that they may be assessed separately according to their level of documentation. With these issues firmly in mind, we make an urgent call for more data on Paraguayan xenarthrans to be collated and for this data to be collected in a standardised way with a view to complementing and/or improving the effectiveness of conservation assessments and the national environmental legislation. A failure to do so may lead to potentially disastrous consequences for populations of xenarthrans in a region of high diversity and importance for their global conservation.

\section{ACKNOWLEDGEMENTS}

Agustín Abba (CEPAVE), Steffen Bock (Berlin), Jonathan Dunnum (MSB), Jim Patton and Chris J. Conroy (MVZ), Manuel Ruedi (Museum Geneva), Thomas Tietze (Basel), Marie-Dominique Wandhammer (Strasbourg), Paz Sánchez, Karen Colman, and Thomas Vinke offered invaluable assistance with the preparation of this manuscript. Isabel Gamarra de Fox (Curator of the mammal collection - MNHNPy) and Julio Torres helped checking the specimens housed at the MNHNPy, and we thank Hugo Cabral for his contributions. Karina Atkinson and her museum staff deserve praise for the superb and professional job they are doing of maintaining their collection under difficult circumstances at Para La Tierra. We thank the observers who shared their xenarthran records with us. Dr. Robert Owen provided important comments that greatly improved the manuscript. A huge thanks to Bolívar Garcete-Barrett (MNHNPy) who made the 
maps in the paper and reviewed all the coordinates included in the Supplementary Material. The authors would like to add their voices to the clamour for more financial support for the Museo Nacional de Historia Natural del Paraguay, the most important national zoological collection. Agustín Abba, Mariella Superina, and two reviewers also made extremely useful comments on the manuscript.

\section{REFERENCES}

Abba, A.M., S.D. Ríos \& P. Smith. 2017. Xenarthra: armadillos y osos hormigueros. Pp. 41-47 in: Libro Rojo de mamíferos del Paraguay (S. Saldívar, V. Rojas \& D. Giménez, eds.). Asociación Paraguaya de Mastozoología y Secretaría del Ambiente, Asunción, Paraguay.

Abba, A.M. \& M. Superina. 2016. Dasypus hybridus (Cingulata: Dasypodidae). Mammalian Species 48: 1020. https://doi.org/10.1093/mspecies/sew001

Abba, A.M. \& S.F. Vizcaíno. 2008. Los xenartros (Mammalia: Xenarthra) del Museo Argentino de Ciencias Naturales «Bernardino Rivadavia» y del Museo de La Plata, Argentina. Contribuciones del Museo Argentino de Ciencias Naturales Bernardino Rivadavia 4: 5-37.

Acosta, N. \& E. López. 2013. Reservorios mamíferos del Trypanosoma cruzi en Paraguay. Memorias del Instituto de Investigación en Ciencias de la Salud 11: 90-96.

Areskoug, V. 2001. Utilisation of remnant dry-forest corridors by the native fauna in a pastural landscape in the Paraguayan Chaco. CBM:s Skriftserie 3: 25-38.

Azara, F. de. 1801. Essais sur l'histoire naturelle des quadrupèdes de la province du Paraguay. Tome 2. Charles Pougens, Paris, France. 499pp.

Azara, F. de. 1802. Apuntamientos para la historia natural de los quadrúpedos del Paragüay y Río de la Plata. Tomo 2. Imprenta de la Viuda de Ibarra, Madrid, Spain. 328pp.

Benítez, L.G. 1986. Breve historia de grandes hombres. Ediciones Comuneros, Asunción, Paraguay. 390 pp.

Bertassoni, A. 2018. Family Myrmecophagidae (anteaters). Pp. 74-90 in: Handbook of the mammals of the world - volume 8: insectivores, sloths and colugos (D.E. Wilson \& R.A. Mittermeier, eds.). Lynx Edicions, Barcelona.

Bertoni, A. de W. 1914. Fauna Paraguaya. Catálogos sistemáticos de los vertebrados del Paraguay. Gráfica M. Brossa, Asunción, Paraguay. 86 pp.

Bertoni, A. de W. 1939. Catálogos sistemáticos de los vertebrados del Paraguay. Revista de la Sociedad Científica del Paraguay 4: 3-60.

Brooks, D.M. 1995. Distribution and limiting factors of edentates in the Paraguayan Chaco. Edentata 2: $10-15$.
Burmeister, H. 1863. Ein neuer Chlamyphorus. Abhandlungen der Senckenbergischen Naturforschenden Gesellschaft 7: 165-171.

Cabrera, A. 1912. Catálogo metódico de las colecciones de mamíferos del Museo de Ciencias Naturales de Madrid. Trabajos del Museo de Ciencias Naturales 7: 1-147.

Cabrera, A. 1958. Catálogo de los mamíferos de América del Sur. Revista del Museo Argentino de Ciencias Naturales Bernardino Rivadavia, Zoología 4: 1-307.

Cadogan, L. 1992. Diccionario mbyá guaraní-castellano. Biblioteca Paraguaya de Antropología, Volumen 17. Asunción, Paraguay. 200 pp.

Cartes, J.L. 2007. Patrones de uso de los mamíferos del Paraguay: Importancia sociocultural y económica. Pp. 167-186 in: Biodiversidad del Paraguay: una aproximación a sus realidades (D.A. Salas-Dueñas \& J.F. Facetti, eds.). Fundación Moisés Bertoni, Asunción, Paraguay.

Centrón, S., R. Zárate, A. Chivugui, M. Achipurangui, E. Mayeregger \& J.L. Cartes. 2013. Patrones de uso de la fauna en la Reserva de Patrimonio Aché de Kuetuvy, Canindeyú. Paraquaria Natural 1: 25-31.

Chase Sardi, M. 1983. Pequeño Decamerón Nivaclé: literatura oral de una etnia del Chaco Paraguayo. Suplemento Antropológico 18: 15-252.

de la Sancha, N., C. López-González. G. D'Elia, P. Myers, L. Váldez \& M.L. Ortíz. 2017. An annotated checklist of the mammals of Paraguay. Therya 8: 241-260. https://doi.org/10.12933/therya-17-473

Desmarest, A.G. 1804. Tableau méthodique des mammifères. Pp. 5-58 in: Nouveau dictionnaire d'histoire naturelle, appliquée aux arts, principalement à l'agriculture et à l'économie rurale et domestique: par une société de naturalistes et d'agriculteurs: avec des figures tirées des trois règnes de la nature. Chez Deterville, Paris.

Dobrizhoffer, M. 1784. Historia de Abiponibus equestri, bellicosaque Paraguariae natione, Tomus 1. Josephi Nob. De Kurzbek, Viennae, Austria.

Epp, N. 2018. Die Natur des paraguayischen Chaco. Filadelfia, Paraguay. 217 pp.

Erxleben, J.C.P. 1777. Systema regni animalis per classes, ordines, genera, species, varietates cun synonimia et historial animalium: classis 1 Mammalia. Weygandianus, Leipzig, Germany.

Esquivel, E. 2001. Mamíferos de la Reserva Natural del Bosque Mbaracayú, Paraguay. Fundación Moisés Bertoni, Asunción, Paraguay. 87 pp.

Fariña, R. \& T. Hostettler. 2003. Vertebrados de la Reserva Natural del Bosque Mbaracayú. Checklist. Fundación Moisés Bertoni, Asunción, Paraguay. 66 pp.

Fast Schartner, H. 2004. Guía ecológica de la cuenca alta del Riacho Yakaré Sur. Fundación para el Desarrollo Sustentable del Chaco, Loma Plata, Paraguay. 112 pp. 
FAUNA Paraguay. 2018. List of the mammals of Paraguay. http://www.faunaparaguay.com/listmammals.html. Downloaded on 5 November 2018.

Feijó, A., B.D. Patterson \& P. Cordero-Estrela. 2018. Taxonomic revision of the long-nosed armadillos, Genus Dasypus Linnaeus, 1758 (Mammalia, Cingulata). PLoS ONE 13(4): 1-69. https://doi.org/10.1371/ journal.pone.0195084

Frutos, S.D. \& R. A. Van den Bussche. 2002. Genetic diversity and gene flow in nine-banded armadillos in Paraguay. Journal of Mammalogy 83: 815-823. https:// doi.org/10.1644/1545-1542(2002)083<0815:GDAG$\mathrm{FI}>2.0 . \mathrm{CO} ; 2$

Fujita, O., L. Sanabria, A. Inchaustti, A.R. De Arias, Y. Tomizawa \& Y. Oku. 1994. Animal reservoirs for Trypanosoma cruzi infection in an endemic area in Paraguay. Journal of Veterinary Medical Science 56: 305-308. https://doi.org/10.1292/jvms.56.305

Fujita, O., A. Abe, Y. Oku, L. Sanabria, A. Inchaustti \& M. Kamiya. 1995. Nematodes of armadillos in Paraguay: A description of a new species Aspidodera esperanzae (Nematoda: Aspidoderidae). Journal of Parasitology 81: 936-941. https://doi.org/10.2307/3284045

Gamarra de Fox, I. \& A.J. Martin. 1996. Mastozoología. Lista de mamíferos del Paraguay. Pp. 469-573 in: Colecciones de flora y fauna del Museo Nacional de Historia Natural del Paraguay (O. Romero Martínez, ed.). Museo Nacional de Historia Natural del Paraguay, Ministerio de Agricultura y Ganadería. San Lorenzo, Paraguay.

Gamarra de Fox I., J. Van Humbeck, W. Sosa, R. Penayo \& R. Villalba. 1998. Mamíferos. Pp. 55-69 in: Fauna amenazada del Paraguay (Dirección de Parques Nacionales y Vida Silvestre, ed.). Ministerio de Agricultura y Ganadería/Fundación Moisés Bertoni, Asunción, Paraguay.

Gardner, A.L. 2008. Magnorder Xenarthra. Pp. 127-176 in: Mammals of South America. Volume 1: Marsupials, xenarthrans, shrews, and bats (A.L. Gardner, ed.). The University of Chicago Press, Chicago.

GAT \& OPIT. 2014. El territorio Ayoreo Totobiegosode. Patrimonio Natural y Cultural, Alto Paraguay, Chaco Paraguayo. Gente Ambiente y Territorio - Organización Payipie Ichadie Totobiegosode, Asunción, Paraguay. $96 \mathrm{pp}$.

Gatti, C. 1985. Enciclopedia Guaraní-Castellano de ciencias naturales y conocimientos paraguayos. Arte Nuevo Editores, Asunción, Paraguay. 331 pp.

Gibb, G.C. et al. 2016. Shotgun mitogenomics provides a reference phylogenetic framework and timescale for living xenarthrans. Molecular Biology and Evolution 33: 621-642. https://doi.org/10.1093/molbev/ msv250

Gray, J.E. 1865. Revision of the genera and species of entomophagous Edentata, founded on the examination of the specimens in the British Museum. Proceedings of Zoological Society of London 33:
359-386. https://doi.org/10.1111/j.1469-7998.1865. tb02351.x

Gray, J.E. 1869. Catalogue of the carnivorous, pachydermatous and edentate mammalia in the British Museum. British Museum of Natural History, London, UK.

Gray, J.E. 1874. On the short-tailed armadillo Muletia septemcincta. Proceedings of Zoological Society of London 42:244-246.https://doi.org/10.1111/j.10963642.1874.tb02479.x

Guyra Paraguay. 2005. Atlas de las aves del Paraguay. Guyra Paraguay, Asunción, Paraguay. 212 pp.

Hamlett, G.W.D. 1939. Identity of Dasypus septemcinctus Linnaeus with notes on some related species. Journal of Mammalogy 20: 328-336. https://doi. org $/ 10.2307 / 1374259$

Hill, K., G. McMillan \& R. Fariña. 2003. Hunting-related changes in game encounter rates from 1994 to 2001 in the Mbaracayú Reserve, Paraguay. Conservation Biology 17: 1312-1323. https://doi. org/10.1046/j.1523-1739.2003.01135.x

Holmberg, E.L. 1895. La fauna argentina. Pp. 477-577 in: Segundo censo nacional (D.G. de la Fuente, ed.). Taller Tip, Penitenciaría Nacional, Buenos Aires, Argentina.

Hutterer, R. \& G. Peters. 2010. Type specimens of mammals (Mammalia) in the collections of the Zoologisches Forschungsmuseum Alexander Koenig, Bonn. Bonn Zoological Bulletin 59: 3-27.

Itaipú Binacional. 2010. Animales del zoológico de Itaipú. Dirección de Educación Ambiental, Itaipú, Hernandarias, Paraguay. 38 pp.

Kerr, R. 1792. The animal kingdom or zoological system of the celebrated Sir Charles Linnaeus. Class I. Mammalia: containing a complete systematic description, arrangement and nomenclature of all the known species and varieties of the Mammalia, or animals which give suck to their young; being a translation of that part of the Systema Naturae as lately published with great improvements by Professor Gmelin of Goettingen. Together with numerous additions from more recent zoological writers and illustrated with copperplates. A. Strahan, T. Cadell \& W. Creech, Edinburgh, Scotland.

Kerr, J. G. 1901. On the birds observed during a second zoological expedition to the Gran Chaco. Ibis 8: 215-236. https://doi.org/10.1111/j.1474-919X.1901. tb00465.x

Linnaeus, C. 1758. Systema naturae per regna tria naturae, secundum classes, ordines, genera, species cum characteribus, diferentiis, synonymis, locis. editio decima. Laurentii Salvii, Holmiae, Sweden.

Lönnberg, E. 1928. Notes on some South American edentates. Arkiv för Zoologi 20A: 17-25.

Lowen, J.C., L. Bartrina, R.P. Clay \& J.A. Tobias. 1996. Biological surveys and conservation priorities in eastern Paraguay. CSB Conservation Publications, Cambridge, UK. 190 pp. 
Masi Pallarés, R. 2011. Vertebrados del Paraguay. Descripción y hábitos con las denominaciones en Guaraní. Smart C3, Asunción, Paraguay.

Masi Pallarés, R. \& C.A. Benítez Usher. 1982. De la distribución de Ixodina (Van der Hammen, 1968) en el Paraguay. Revista Paraguaya de Microbiología 17: 49-52.

McDonough, C.M. \& W.J. Loughry. 2018. Family Dasypodidae (long-nosed armadillos). Pp. 30-47 in: Handbook of the mammals of the world - volume 8: insectivores, sloths and colugos (D. E. Wilson \& R. A. Mittermeier, eds.). Lynx Edicions, Barcelona.

Mereles, F. 2013. Acerca de las extensiones de Cerrados y Cerradones en el Paraguay. Paraquaria Natural 1: 35-38.

Meritt, D. A. Jr. 1973. Observations on the status of the giant armadillo, Priodontes giganteus, in Paraguay. Zoologica 58: 103.

Meritt, D.A. Jr. 2008. Xenarthrans of the Paraguayan Chaco. Pp. 294-299 in: The biology of the Xenarthra (S.F. Vizcaíno \& W.J. Loughry, eds.). University Press of Florida, Gainesville.

Mertens, R. 1925. Verzeichnis der Säugetier-Typen des Senckenbergischen Museums. Senckenbergiana 7: 18-37.

Morales, M. 2007. Diversidad de mamíferos en Paraguay. Pp. 133-150 in: Biodiversidad del Paraguay: una aproximación a sus realidades (D.A. Salas-Dueñas \& J.F. Facetti, eds.). Fundación Moisés Bertoni, Asunción, Paraguay.

Myers, P. \& R. M. Wetzel. 1979. New records of mammals from Paraguay. Journal of Mammalogy 60: 638-641. https://doi.org/10.2307/1380113

Nava, S. et al. 2007. The ticks (Acari: Ixodida: Argasidae, Ixodidae) of Paraguay. Annals of Tropical Medicine and Parasitology 101: 255-270. https://doi. org/10.1179/136485907X176319

Neris, N. \& F. Colman. 2001. Mamíferos. Pp. 127-247 in: Especies silvestres del Paraguay. Guía de identificación de especies con importancia económica. Secretaría Nacional del Medio Ambiente-Prodechaco-Guyra Paraguay, Asunción, Paraguay.

Neris, N., F. Colman, E. Ovelar, N. Sukigara \& N. Ishii. 2002. Guía de mamíferos medianos y grandes del Paraguay: distribución, tendencia poblacional y utilización. SEAM, Asunción, Paraguay. 165 pp.

Neris, N. \& E.G. Franco Rivarola. 2005. Mamíferos. Pp. 153-193 in: Guía ilustrada de anfibios, reptiles, aves y mamíferos del Paraguay (N. Neris, ed.). Natura Vita - Jica, Asunción, Paraguay.

Olson, D. M. et al. 2001. Terrestrial ecoregions of the world: a new map of life on Earth. BioScience 51: 933-938. https://doi.org/10.1641/0006-3568(2001) 051[0933:TEOTWA]2.0.CO;2

Quijada, B. 1910. Catálogo de los vertebrados vivientes conservados en el Museo Nacional de Chile. Boletín del Museo Nacional de Chile 1: 89-132.
Ramírez Pinto, F. \& M.C. Velázquez. 2010. Mamíferos. Pp. 23-25 in: La naturaleza y riqueza del río Pilcomayo (L. Villalba, ed.). Iniciativa Chaco Trinacional y Fundación Moisés Bertoni, Asunción, Paraguay. 35 pp.

Redford, K.H. 1985. Food habits of armadillos (Xenarthra: Dasypodidae). Pp. 429-427 in: The evolution and ecology of armadillos, sloths and vermilinguas (G.G. Montgomery, ed.). Smithsonian Institution Press, Washington D.C.

Rengger, J.R. 1830. Naturgeschichte der Saeugethiere von Paraguay. Schweighausersche Buchhandlung, Basel, Switzerland. 394 pp. https://doi.org/10.5962/ bhl.title.49975

Roguin, L. de. 1986. Les mammifères du Paraguay dans les collections du Muséum de Genève. Revue Suisse de Zoologie 93: 1009-1022. https://doi. org/10.5962/bhl.part.79525

Saldívar, S. et al. 2017. Los mamíferos amenazados del Paraguay. Pp. 25-32 in: Libro Rojo de mamíferos del Paraguay (S. Saldívar, V. Rojas \& D. Giménez, eds.). Asociación Paraguaya de Mastozoología y Secretaría del Ambiente, Asunción, Paraguay.

Sanborn, C.C. 1930. Distribution and habits of the three-banded armadillo Tolypeutes. Journal of Mammalogy 11: 61-68. https://doi.org/10.2307/1373787

Sánchez Labrador, J. 1910. El Paraguay católico. Homenaje de la Universidad Nacional de La Plata al XVII Congreso Internacional de los Americanistas en su reunión de Buenos Aires, en mayo 16 a 21 de 1910. Buenos Aires, Argentina.

Schinz, H.R. 1825. Das Thierreich eingetheilt nach dem Bau der Thiere als Grundlage ihrer Naturgeschichte und der vergleichenden Anatomie. Vol. 4. J.G. Cotta, Stuttgart and Tübingen, Germany.

Schmidt, G.D. \& R.L. Martin. 1978. Tapeworms of Chaco Boreal, Paraguay, with two new species. Journal of Helminthology 52: 205-209. https://doi. org/10.1017/S0022149X00005381

Seelwische, J. 1980. Diccionario Nivacle-Castellano. Vol. 1. Biblioteca Paraguaya de Antropología, Mariscal Estigarribia. 378 pp.

Servicio Forestal Nacional. 1982. Guía de algunos vertebrados del Parque Nacional Ybycuí. Ministerio de Agricultura y Ganadería, Asunción, Paraguay. 47 pp.

Smales, L.R. 2007. Oligacanthorhynchidae (Acanthocephala) from mammals from Paraguay with the description of a new species of Neoncicola. Comparative Parasitology 74: 237-243. https://doi. org/10.1654/4271.1

Smith, P. 2007a. Giant anteater Myrmecophaga tridactyla. FAUNA Paraguay Handbook of the mammals of Paraguay 2: 1-18. Update April 2012. http://www.faunaparaguay.com/mamm2Myrmecophagatridactyla.pdf

Smith, P. 2007b. Giant armadillo Priodontes maximus. FAUNA Paraguay Handbook of the mammals of Paraguay 6: 1-11. Update April 2012. http://www.faunaparaguay.com/mamm6Priodontesmaximus.pdf 
Smith, P. 2007c. Nine-banded armadillo Dasypus novemcinctus. FAUNA Paraguay Handbook of the mammals of Paraguay 8: 1-30. Update April 2012. http:// www.faunaparaguay.com/mamm8Dasypusnovemcinctus.pdf

Smith, P. 2007d. Six-banded armadillo Euphractus sexcinctus. FAUNA Paraguay Handbook of the mammals of Paraguay 5:1-16. Update April 2012.http://www.faunaparaguay.com/mamm5Euphractussexcinctus.pdf

Smith, P. 2007e. Southern tamandua Tamandua tetradactyla. FAUNA Paraguay Handbook of the mammals of Paraguay 3: 1-15. Update April 2012. http:// www.faunaparaguay.com/mamm3Tamanduatetradactyla.pdf

Smith, P. 2007f. Southern three-banded armadillo Tolypeutes matacus. FAUNA Paraguay Handbook of the mammals of Paraguay 7: 1-12. Update April 2012. http://www.faunaparaguay.com/mamm7Tolypeutesmatacus.pdf

Smith, P. 2008a. Chaco fairy armadillo Calyptophractus retusus. FAUNA Paraguay Handbook of the mammals of Paraguay 20: 1-5. http://www.faunaparaguay. com/mamm20Calyptophractusretusus.pdf

Smith, P. 2008b. Greater hairy armadillo Chaetophractus villosus. FAUNA Paraguay Handbook of the mammals of Paraguay 11: 1-15. Update April 2012. http://www.faunaparaguay.com/mamm11Chaetophractusvillosus.pdf

Smith, P. 2008c. Greater naked-tailed armadillo Cabassous tatouay. FAUNA Paraguay Handbook of the mammals of Paraguay 13: 1-9. Update April 2012. http:// www.faunaparaguay.com/mamm13Cabassoustatouay.pdf

Smith, P. 2008d. Lesser hairy armadillo Chaetophractus vellerosus. FAUNA Paraguay Handbook of the mammals of Paraguay 12: 1-9. Update February 2011. http://www.faunaparaguay.com/mamm12Chaetophractusvellerosus.pdf

Smith, P. 2008e. Seven-banded armadillo Dasypus septemcinctus. FAUNA Paraguay Handbook of the mammals of Paraguay 14: 1-7. Update February 2011. http://www.faunaparaguay.com/mamm14Dasypusseptemcinctus.pdf

Smith, P. 2008f. Southern long-nosed armadillo Dasypus hybridus. FAUNA Paraguay Handbook of the mammals of Paraguay 15: 1-10. Update February 2011. http://www.faunaparaguay.com/mamm15Dasypushybridus.pdf

Smith, P. 2009. Chaco naked-tailed armadillo Cabassous chacoensis. FAUNA Paraguay Handbook of the mammals of Paraguay 25: 1-7. Update April 2012. http:// www.faunaparaguay.com/mamm 25Cabassouschacoensis.pdf

Smith, P. 2011. Southern naked-tailed armadillo Cabassous unicinctus. FAUNA Paraguay Handbook of the mammals of Paraguay 40: 1-10. Update April 2012. http://www.faunaparaguay.com/mamm40Cabassousunicinctus.pdf
Smith, P. 2012. Assessing the assessment, the relevance of the 2006 Paraguayan mammal Red List to the reality of Xenarthra conservation in 2012. Edentata 13: 18-28. https://doi.org/10.5537/020.013.0101

Smith, P. \& R.D. Owen. 2017. Calyptophractus retusus (Cingulata: Dasypodidae). Mammalian Species 49(947): 57-62. https://doi.org/10.1093/mspecies/sex005

Smith, P., R.D. Owen, K. Atkinson, H. del Castillo \& E. Northcote-Smith. 2011. First records of the southern naked-tailed armadillo Cabassous unicinctus (Linnaeus, 1758) (Xenarthra: Dasypodidae) in Paraguay. Edentata 12: 53-57. https://doi.org/10.5537/020.013.0101

Superina, M. \& A.M. Abba. 2018. Family Chlamyphoridae (Chlamyphorid armadillos). Pp. 48-73 in: Handbook of the mammals of the world - volume 8: insectivores, sloths and colugos (D.E. Wilson \& R.A. Mittermeier, eds.). Lynx Edicions, Barcelona.

Superina, M., T. Plese, N. Moraes-Barros \& A.M. Abba. 2010. The 2010 sloth Red List assessment. Edentata 11: $115-134$.

Thomas, O. 1907. On Neotropical mammals of the genera Callicebus, Reithrodontomys, Ctenomys, Dasypus and Marmosa. Annals and Magazine of Natural History Series 7 20: 161-168. https://doi. org/10.1080/00222930709487318

Thomas, O. 1911. The mammals of the tenth edition of Linnaeus; an attempt to fix the types of the genera and the exact bases and localities of the species. Proceedings of the Zoological Society of London 79: 120-158.

Unruh, E. \& H. Kalisch. 1997. Moya'ansaeclha' Nengelpayvaam Nengeltomha Enlhet. Biblioteca Paraguaya de Antropología, Vol. 27. Centro de Estudios Antropológicos de la Universidad Católica (CEADUC), Asunción. 903 pp.

Velázquez, M. \& F. Ramírez Pinto. 2014. Guía de los mamíferos de la Reserva Natural Tapytá. Fundación Moisés Bertoni, Asunción, Paraguay. 127 pp.

Villalba R. \& A. Yanosky. 2000. Guía de huellas y señales: fauna paraguaya. Fundación Moisés Bertoni, Asunción, Paraguay. 112 pp.

Vinke, T. \& S. Vinke. 2008. El maravilloso Chaco Paraguayo. Dauri-Verlag, Bergheim, Germany. 96 pp.

Vinke, T. \& S. Vinke. 2014. El pichiciego mayor, una rareza poco conocida de la tierra chaqueña Chlamyphorus retusus. Paraguay Salvaje 2: 8-11.

Weiler, A. \& K. Nuñez. 2012. Desafíos para la conservación del tatú carreta (Priodontes maximus) en el Chaco Paraguayo. Reportes Científicos de la FACEN 3: $5-13$.

Wetmore, A. 1926. Observations on the birds of Argentina, Paraguay, Uruguay and Chile. Bulletin of the United States National Museum 133: 1-448. https:// doi.org/10.5479/si.03629236.133.i

Wetzel, R.M. 1977. The Chacoan peccary Catagonus wagneri (Rusconi). Bulletin of the Carnegie Museum of Natural History 3: 1-36. 
Wetzel, R.M. 1980. Revision of the naked-tailed armadillos, genus Cabassous McMurtrie. Annals of the Carnegie Museum of Natural History 49: 323-357.

Wetzel, R. 1985a. Taxonomy and distribution of armadillos. Pp. 23-46 in: The evolution and ecology of armadillos, sloths and vermilinguas (G.G. Montgomery, ed.). Smithsonian Institution Press, Washington D.C.

Wetzel, R. 1985b. The identification and distribution of recent Xenarthra (= Edentata). Pp. 5-22 in: The evolution and ecology of armadillos, sloths and vermilinguas (G.G. Montgomery, ed.). Smithsonian Institution Press, Washington D.C.

Wetzel, R.M. \& J.W. Lovett. 1974. A collection of mammals from the Chaco of Paraguay. The University of Connecticut Occasional Papers: Biological Science, Series 2: 203-216.

Wetzel, R.M. \& E. Mondolfi. 1979. The subgenera and species of long-nosed armadillos, Genus Dasypus L. Pp. 43-63 in: Vertebrate ecology in the northern Neotropics (J.F. Eisenberg, ed.). Smithsonian Institution Press, Washington D.C.

Wetzel, R. M., A. L. Gardner, K. H. Redford \& J.F. Eisenberg. 2008. Order Cingulata. Pp. 128-156 in: Mammals of South America, Volume 1: marsupials, xenarthrans, shrews and bats (A.L. Gardner, ed.). The University of Chicago Press, Chicago.

Wilson D.E. \& D.M. Reeder. 2005. Mammal species of the world. A taxonomic and geographic reference ( $3^{\text {rd }}$ ed.). The Johns Hopkins University Press, Baltimore. 2142 pp.
Yahnke C. J., I. Gamarra de Fox \& F. Colman. 1998. Mammalian species richness in Paraguay: the effectiveness of national parks in preserving biodiversity. Biological Conservation 84: 263-268. https://doi. org/10.1016/S0006-3207(97)00113-4

Yeo, M. et al. 2005. Origins of Chagas disease: Didelphis species are natural hosts of Trypanosoma cruzi I and armadillos hosts of Trypanosoma cruzi II, including hybrids. International Journal for Parasitology 35: 225233. https://doi.org/10.1016/j.ijpara.2004.10.024

Yepes, J. 1928. Los «Edentata» argentinos. Sistemática y distribución. Revista de la Universidad de Buenos Aires Serie 2a 1: 461-515.

Yepes, J. 1933. Una especie nueva de «mulita» (Dasipodinae) para el norte argentino. Physis 11: 22-232.

Yepes, J. 1935. Las especies argentinas del género Cabassous (Dasypodidae). Physis 11: 438-444.

Yepes, J. 1939. Una nueva subespecie de pichi ciego mayor (Chlamyphorinae) y su probable distribución geográfica. Physis 16: 35-39.

Ziegler, T., J. Unger, A. Feiler \& E. Lehr. 2002. The first Gran Chaco expedition of the Museum für Tierkunde Dresden: records of amphibians, reptiles and mammals from the Dry Chaco of Paraguay (Amphibia, Reptilia, Mammalia). Faunistische Abhandlungen Staatliches Museum für Tierkunde Dresden 23: $219-238$.

Received: 9 November 2018; Accepted: 7 December 2018 